\title{
Discrete spectrum of quantum Hall effect Hamiltonians I. Monotone edge potentials
}

\author{
Vincent Bruneau, Pablo Miranda, and Georgi Raikov
}

\begin{abstract}
We consider the unperturbed operator $H_{0}=(-i \nabla-\mathbf{A})^{2}+W$, self-adjoint in $L^{2}\left(\mathbb{R}^{2}\right)$. Here $\mathbf{A}$ is a magnetic potential which generates a constant magnetic field $b>0$, and the edge potential $W$ is a non-decreasing non-constant bounded function depending only on the first coordinate $x \in \mathbb{R}$ of $(x, y) \in \mathbb{R}^{2}$. Then the spectrum of $H_{0}$ has a band structure and is absolutely continuous; moreover, the assumption $\lim _{x \rightarrow \infty}(W(x)-W(-x))<2 b$ implies the existence of infinitely many spectral gaps for $H_{0}$. We consider the perturbed operators $H_{ \pm}=H_{0} \pm V$ where the electric potential $V \in L^{\infty}\left(\mathbb{R}^{2}\right)$ is non-negative and decays at infinity. We investigate the asymptotic distribution of the discrete spectrum of $H_{ \pm}$in the spectral gaps of $H_{0}$. We introduce an effective Hamiltonian which governs the main asymptotic term; this Hamiltonian involves a pseudo-differential operator with generalized anti-Wick symbol equal to $V$. Further, we restrict our attention on perturbations $V$ of compact support and constant sign. We establish a geometric condition on the support of $V$ which guarantees the finiteness of the number of the eigenvalues of $H_{ \pm}$in any spectral gap of $H_{0}$. In the case where this condition is violated, we show that, generically, the convergence of the infinite series of eigenvalues of $H_{+}$(resp. $H_{-}$) to the lower (resp. upper) edge of a given spectral gap, is Gaussian.
\end{abstract}

Mathematics Subject Classification (2010). 35P20, 35J10, 47F05, 81Q10.

Keywords. Magnetic Schrödinger operators, spectral gaps, eigenvalue distribution.

\section{Contents}

1 Introduction . . . . . . . . . . . . . . . . . . 238

2 Basic spectral properties of $H_{0} \ldots \ldots \ldots \ldots \ldots \ldots \ldots \ldots$

3 Effective Hamiltonians . . . . . . . . . . . . . . . . . . . 247

4 Sufficient condition for the boundedness of $\mathcal{N}_{j}^{+}(\lambda) \ldots \ldots \ldots . \ldots 252$

5 Reduction to operators in holomorphic spaces . . . . . . . . . . 254

6 Asymptotic bounds of $\mathcal{N}_{j}^{+}(\lambda)$ as $\lambda \downarrow 0 \ldots \ldots \ldots \ldots \ldots$

6.1 Lower bound of $\mathcal{N}_{j}^{+}(\lambda) \ldots \ldots \ldots \ldots \ldots \ldots$

6.2 Upper bound of $\mathcal{N}_{j}^{+}(\lambda) \ldots \ldots \ldots \ldots \ldots \ldots \ldots$

References . . . . . . . . . . . . . . . . . . . . . 270 


\section{Introduction}

The general form of the unperturbed operators we are going to consider in the present article and its successor [16], is

$$
H_{0}=H_{0}(b, W)=-\frac{\partial^{2}}{\partial x^{2}}+\left(-i \frac{\partial}{\partial y}-b x\right)^{2}+W(x) .
$$

Here $b>0$ is the constant magnetic field, and the edge potential $W \in L^{\infty}(\mathbb{R} ; \mathbb{R})$ is independent of $y$. The self-adjoint operator $H_{0}$ is defined initially on $C_{0}^{\infty}\left(\mathbb{R}^{2}\right)$ and then is closed in $L^{2}\left(\mathbb{R}^{2}\right)$. Let $\mathcal{F}$ be the partial Fourier transform with respect to $y$, i.e.

$$
(\mathcal{F} u)(x, k)=(2 \pi)^{-1 / 2} \int_{\mathbb{R}} e^{-i k y} u(x, y) d y, \quad(x, k) \in \mathbb{R}^{2} .
$$

Then we have

$$
\widetilde{F} H_{0} \mathcal{F}^{*}=\int_{\mathbb{R}}^{\oplus} h(k) d k
$$

where the operator

$$
h(k)=-\frac{d^{2}}{d x^{2}}+(b x-k)^{2}+W(x), \quad k \in \mathbb{R},
$$

is self-adjoint in $L^{2}(\mathbb{R})$. Note that $h(k), k \in \mathbb{R}$, is a Kato analytic family (see [13] or [25], Section XII.2). For $w \in L^{2}(\mathbb{R})$ and $k \in \mathbb{R}$ set $\left(\tau_{k} w\right)(x)=\underset{\sim}{w}(x-k / b)$. Evidently $\tau_{k}$ is a unitary operator in $L^{2}(\mathbb{R})$, and we have $\tau_{k}^{*} h(k) \tau_{k}=\tilde{h}(k)$ where

$$
\tilde{h}(k)=-\frac{d^{2}}{d x^{2}}+b^{2} x^{2}+W(x+k / b), \quad k \in \mathbb{R} .
$$

Evidently, for each $k \in \mathbb{R}$ the operator $h(k)$ (and, hence, $\tilde{h}(k)$ ) has a discrete and simple spectrum. Let $\left\{E_{j}(k)\right\}_{j=1}^{\infty}$ be the increasing sequence of the eigenvalues of $h(k)$ (and, hence, of $\tilde{h}(k)$ ). The Kato analytic perturbation theory [13] implies that $E_{j}(k), j \in \mathbb{N}$, are real analytic functions of $k \in \mathbb{R}$. When we need to indicate the dependence of $E_{j}(k)$ on $b$ and/or $W$, we will write $E_{j}(k ; b, W)$ or $E_{j}(k ; W)$ instead of $E_{j}(k)$. Note that if $W=0$, then the eigenvalues are independent of $k$, and their explicit form is well known:

$$
E_{j}(k ; b, 0)=E_{j}(b, 0)=b(2 j-1), \quad k \in \mathbb{R}, j \in \mathbb{N} .
$$

Further,

$$
\sigma\left(H_{0}\right)=\bigcup_{j=1}^{\infty} \overline{E_{j}(\mathbb{R})}
$$


In the present article we will consider monotone $W$. For definiteness we assume that $W$ is non-decreasing. Then the band functions $E_{j}, j \in \mathbb{N}$, are also non-decreasing, and $\sigma\left(H_{0}\right)=\bigcup_{j=1}^{\infty}\left[\mathcal{E}_{j}^{-}, \mathcal{E}_{j}^{+}\right]$with

$$
\begin{aligned}
& \mathcal{E}_{j}^{-}=\lim _{k \rightarrow-\infty} E_{j}(k)=b(2 j-1)+W_{-}, \\
& \mathcal{E}_{j}^{+}=\lim _{k \rightarrow \infty} E_{j}(k)=b(2 j-1)+W_{+},
\end{aligned}
$$

and

$$
W_{-}=\lim _{x \rightarrow-\infty} W(x), \quad W_{+}=\lim _{x \rightarrow \infty} W(x)
$$

(see Proposition 2.1 below). Throughout the article we assume that

$$
W_{-}<W_{+},
$$

i.e. $W$ is not identically constant. Hence, $\mathcal{E}_{j}^{-}<\mathcal{E}_{j}^{+}$for each $j \in \mathbb{N}$. Moreover, we will assume that

$$
W_{+}-W_{-}<2 b \text {. }
$$

Then we have

$$
\varepsilon_{j}^{+}<\varepsilon_{j+1}^{-}, \quad j \in \mathbb{N},
$$

and the intervals $\left(\mathcal{E}_{j}^{+}, \mathcal{E}_{j+1}^{-}\right), j \in \mathbb{N}$, are open gaps in the spectrum of $H_{0}$. Thus all the bands in the spectrum of $H_{0}$ are separated by gaps where discrete spectrum may appear under appropriate perturbations.

The perturbations under consideration will be electric potentials $V: \mathbb{R}^{2} \rightarrow \mathbb{R}$ which are $\Delta$-compact. A simple sufficient condition which guarantees the compactness of the operator $V(-\Delta-i)^{-1}$, is

$$
V \in L_{0}^{\infty}\left(\mathbb{R}^{2}\right)=\left\{u \in L^{\infty}\left(\mathbb{R}^{2}\right) \mid u(x, y) \rightarrow 0 \text { as } x^{2}+y^{2} \rightarrow \infty\right\} .
$$

By the diamagnetic inequality, the operator $V\left(H_{0}-i\right)^{-1}$ is also compact, and hence

$$
\sigma_{\mathrm{ess}}\left(H_{0}+V\right)=\sigma_{\mathrm{ess}}\left(H_{0}\right)=\bigcup_{j=1}^{\infty}\left[\mathcal{E}_{j}^{-}, \mathcal{E}_{j}^{+}\right] .
$$

For simplicity, we will consider perturbations of definite sign. More precisely we will suppose that $V \geq 0$, and will consider the operators $H_{ \pm}=H_{0} \pm V$. Note that in the case of positive (resp. negative) perturbations, the discrete eigenvalues of the perturbed operator which may appear in a given open gap of the spectrum of the unperturbed operator, may accumulate only to the lower (resp. upper) edge of the gap.

In order to give a more explicit formulation of the problem, we need the following notations. Let $T$ be a self-adjoint linear operator in a Hilbert space. Denote by $\mathbb{P}_{\mathcal{O}}(T)$ the spectral projection of $T$ corresponding to the Borel set $\mathcal{O} \subseteq \mathbb{R}$. For $\lambda>0$ set

$$
\mathcal{N}_{0}^{-}(\lambda)=\operatorname{rank} \mathbb{P}_{\left(-\infty, \mathcal{E}_{1}^{-}-\lambda\right)}\left(H_{-}\right) .
$$


Next, fix $j \in \mathbb{N}$ and assume that (1.4) holds. Pick $\lambda \in\left(0, \mathcal{E}_{j+1}^{-}-\mathcal{E}_{j}^{+}\right)$, and set

$$
\mathcal{N}_{j}^{-}(\lambda)=\operatorname{rank} \mathbb{P}_{\left(\mathcal{E}_{j}^{+}, \mathcal{E}_{j+1}^{-}-\lambda\right)}\left(H_{-}\right), \quad \mathcal{N}_{j}^{+}(\lambda)=\operatorname{rank} \mathbb{P}_{\left(\mathcal{E}_{j}^{+}+\lambda, \mathcal{E}_{j+1}^{-}\right)}\left(H_{+}\right) .
$$

We reduce the investigation of the accumulation of the discrete eigenvalues to the edges of the gap $\left(\mathcal{E}_{j}^{+}, \mathcal{E}_{j+1}^{-}\right)$of its essential spectrum, to the study of the asymptotic behavior as $\lambda \downarrow 0$ of the counting functions $\mathcal{N}_{j}^{ \pm}(\lambda)$.

The investigation of the asymptotic behavior of the discrete spectrum of perturbed analytically fibred quantum Hamiltonians, lying in the gaps of the essential one has a long history. Probably, the first results of this type were obtained for the Schrödinger operator with periodic potential perturbed by a decaying one (see, e.g., [29], [14], [21], and [27]). Recently, similar problems have been considered for perturbed 2D magnetic Hamiltonians [4], and for Dirichlet Laplacians in twisted wave-guides [3]. The common feature of the above cited articles is that the edges of the gaps in the spectrum of the unperturbed operator coincide with the extremal values of the band functions taken at local non degenerate extrema; in this case the arising effective Hamiltonian is a differential Schrödinger type operator. In the present article the edges of the gaps are the limiting values of the band functions $E_{j}(k)$ as $k \rightarrow \pm \infty$. The effective Hamiltonian which arises in this case involves a "kinetic" part equal in the momentum representation to the multiplier by $E_{j}$, and a "potential" part which is a pseudodifferential operator ( $\Psi D O)$ with contravariant (generalized anti-Wick) symbol equal to $V$. These $\Psi$ DOs are unitarily equivalent to the BerezinToeplitz operators which appear as effective Hamiltonians in the study of compact perturbations of the Landau operator (see, e.g., [20], and [23]). Note however that in the case of the Landau operator (which is equal to $H_{0}$ with $W=0$ ) the kinetic part of the effective Hamiltonian is proportional to the identity operator.

The article is organized as follows. In Section 2 we describe the basic spectral properties of the unperturbed operators which we need in the sequel. In Section 3 we introduce the effective Hamiltonian appropriate for the asymptotic analysis as $\lambda \downarrow 0$ of the functions $\mathcal{N}_{j}^{ \pm}(\lambda)$ with fixed $j \in \mathbb{N}$. Our effective Hamiltonian approach allows us to consider various types of $W$ and $V$ which satisfy the assumptions stated above. Nonetheless, the rest of the article is dedicated to the case where $V \in L_{0}^{\infty}\left(\mathbb{R}^{2} ; \mathbb{R}\right)$ has a compact support. This choice is motivated by the possible applications in the theory of the quantum Hall effect (see, e.g., [6], [10], and [5]), and, on the other hand, by the spectacular progress in the investigation of the discrete spectrum for localized perturbations of the Landau Hamiltonian $H_{0}(b, 0)$ (see, e.g., [23], [15], [18], [7], [26], [17], and [19]). For definiteness, we suppose that $V \geq 0$ and discuss only the behavior of the counting functions $\mathcal{N}_{j}^{+}(\lambda), j \in \mathbb{N}$, near the lower edges of the spectral gaps; in the case $V \leq 0$ the behavior of $\mathcal{N}_{j}^{-}(\lambda)$ near the upper edges is analogous. In Section 4 we establish a sufficient condition of geometric nature which guarantees that all the functions $\mathcal{N}_{j}^{+}(\lambda), j \in \mathbb{N}$, remain bounded as $\lambda \downarrow 0$, i.e. that there is a finite number of eigenvalues of $H_{+}$in any gap of its essential spectrum. When this sufficient condition is violated, we show that for any $j \in \mathbb{N}$ the functions 
$\mathcal{N}_{j}^{+}(\lambda)$ generically blow up as $\lambda \downarrow 0$. More precisely, in Section 5 we reduce the analysis of $\mathcal{N}_{j}^{+}(\lambda)$ to counting functions for operators in holomorphic spaces. These operators are studied in Section 6 in order to establish a lower asymptotic estimate

$$
e_{-}|\ln \lambda|^{1 / 2}(1+o(1)) \leq \mathcal{N}_{j}^{+}(\lambda), \quad \lambda \downarrow 0, j \in \mathbb{N},
$$

with $\mathcal{C}_{-}>0$ which holds when the sufficient condition of Section 4 is not fulfilled, and an upper asymptotic estimate

$$
\mathcal{N}_{j}^{+}(\lambda) \leq \mathscr{C}_{+}|\ln \lambda|^{1 / 2}(1+o(1)), \quad \lambda \downarrow 0, j \in \mathbb{N},
$$

with $\ell_{+}>\ell_{-}$. Note that the constants $\ell_{ \pm}$in (1.6) and (1.7) admit a clear geometric interpretation and are independent of $j \in \mathbb{N}$. Thus, in the case of infinitely many eigenvalues in any given gap, the main asymptotic term of $\mathcal{N}_{j}^{+}(\lambda)$ is expected to be of order $|\ln \lambda|^{1 / 2}$ which, loosely speaking, corresponds to a Gaussian convergence of the discrete eigenvalues to the edges of the gaps of the essential spectrum. This behavior is different from the case of compactly supported perturbations of the Landau Hamiltonian where typically we have

$$
\mathcal{N}_{j}^{+}(\lambda) \sim \frac{|\ln \lambda|}{\ln |\ln \lambda|}, \lambda \downarrow 0, \quad j \in \mathbb{N},
$$

(see, e.g., [23]). Hopefully, in a future work we will attack the problem of finding the main asymptotic term as $\lambda \downarrow 0$ of $\mathcal{N}_{j}^{ \pm}(\lambda), j \in \mathbb{N}$.

Finally, we note that in the second part [16] of this study, we consider analogous problems for periodic edge potentials $W$.

Acknowledgments. The authors were partially supported by the Chilean Science Foundation Fondecyt under Grant 1090467, and by the Bernoulli Center, EPFL, Lausanne, where a part of this work was done within the framework of the Program "Spectral and Dynamical Properties of Quantum Hamiltonians", January - June 2010.

V. Bruneau was also partially supported by the Agence Nationale de la Recherche under Grant NONAa (ANR-08-BLAN-0228).

P. Miranda and G. Raikov were also partially supported by Núcleo Científico ICM P07-027-F "Mathematical Theory of Quantum and Classical Magnetic Systems".

\section{Basic spectral properties of $\boldsymbol{H}_{0}$}

In the following proposition we consider the general properties of the band functions $E_{j}, j \in \mathbb{N}$. By analogy with the operator $\tilde{h}(k)$, introduce the shifted harmonic oscillator

$$
\tilde{h}_{\infty}=-\frac{d^{2}}{d x^{2}}+b^{2} x^{2}+W_{+},
$$

which is self-adjoint in $L^{2}(\mathbb{R})$, and essentially self-adjoint on $C_{0}^{\infty}(\mathbb{R})$. 
Proposition 2.1. Assume that $W$ is non-decreasing and bounded. Then for each $j \in \mathbb{N}$ the eigenvalue $E_{j}(k)$ is a non-decreasing function of $k \in \mathbb{R}$, and (1.2) holds true.

Proof. The fact that $E_{j}$ are non-decreasing bounded functions of $k$ follows directly from the mini-max principle. Let us prove (1.2). Pick $E>-b-W_{-}$. Then for each $k \in \mathbb{R}$ we have $-E<b+W_{-} \leq \inf \sigma(\tilde{h}(k))$. Moreover, $-E<b+W_{+}=$ $\inf \sigma\left(\tilde{h}_{\infty}\right)$. Then,

$$
\begin{array}{r}
\left|\left(E_{j}(k)+E\right)^{-1}-\left(b(2 j-1)+W_{+}+E\right)^{-1}\right| \leq \\
\left\|(\tilde{h}(k)+E)^{-1}\left(W_{+}-W(\cdot+k / b)\right)\left(\tilde{h}_{\infty}+E\right)^{-1}\right\| \leq \\
\left\|(\tilde{h}(k)+E)^{-1}\right\|\left\|\left(W_{+}-W(\cdot+k / b)\right)\left(\tilde{h}_{\infty}+E\right)^{-1}\right\| .
\end{array}
$$

Moreover,

$$
\left\|(\tilde{h}(k)+E)^{-1}\right\| \leq\left(E+b+W_{-}\right)^{-1},
$$

and the r.h.s. is $k$-independent. Further, the multiplier by $\left(W_{+}-W(\cdot+k / b)\right), x \in \mathbb{R}$, tends strongly to zero as $k \rightarrow \infty$, while the operator $\left(\tilde{h}_{\infty}+E\right)^{-1}$ is compact and $k$-independent. Hence, the operator $\left(W_{+}-W(\cdot+k / b)\right)\left(\tilde{h}_{\infty}+E\right)^{-1}$ tends uniformly to zero as $k \rightarrow \infty$. Now, (2.2)-(2.3) imply

$$
\lim _{k \rightarrow \infty}\left(E_{j}(k)+E\right)^{-1}=\left(b(2 j-1)+W_{+}+E\right)^{-1}, \quad j \in \mathbb{N},
$$

which yields the second limit in (1.2). The first one is proved in the same manner.

Proposition 2.1 implies that if (1.3) holds, then there are no identically constant functions $E_{j}, j \in \mathbb{N}$. Applying the general theory of analytically fibred Hamiltonians (see, e.g., [25], Section XIII.16), we immediately obtain the following result.

Corollary 2.2. Assume that $W$ is increasing and bounded, and that (1.3) holds true. Then the spectrum of the operator $H_{0}$ is absolutely continuous.

Our next theorem will play a crucial role in the construction of the effective Hamiltonian introduced in the next section. For its formulation we need the following notations. Fix $k \in \mathbb{R}$ and $j \in \mathbb{N}$ denote by $\pi_{j}(k)$ the orthogonal projection onto $\operatorname{Ker}\left(h(k)-E_{j}(k)\right)$. Then we have

$$
\pi_{j}(k)=\left\langle\cdot, \psi_{j}(\cdot ; k)\right\rangle \psi_{j}(\cdot ; k),
$$

where $\langle\cdot, \cdot\rangle$ is the scalar product in $L^{2}(\mathbb{R})$, and $\psi_{j}(x ; k), x \in \mathbb{R}$, is an eigenfunction of $h(k)$ which satisfies

$$
h(k) \psi_{j}(\cdot ; k)=E_{j}(k) \psi_{j}(\cdot ; k), \quad\left\|\psi_{j}(\cdot ; k)\right\|_{L^{2}(\mathbb{R})}=1 .
$$

Moreover, $\psi_{j}(\cdot ; k)$ could be chosen to be real-valued. 
Set

$$
h_{\infty}(k)=\tau_{k} \tilde{h}_{\infty} \tau_{k}^{*}=-\frac{d^{2}}{d x^{2}}+(b x-k)^{2}+W_{+}, \quad k \in \mathbb{R},
$$

the operator $\tilde{h}_{\infty}$ being defined in (2.1). Denote by $\pi_{j, \infty}(k), k \in \mathbb{R}, j \in \mathbb{N}$, the orthogonal projection onto $\operatorname{Ker}\left(h_{\infty}(k)-\varepsilon_{j}^{+}\right)$. Then, similarly to (2.4), we have

$$
\pi_{j, \infty}(k)=\left\langle\cdot, \psi_{j, \infty}(\cdot ; k)\right\rangle \psi_{j, \infty}(\cdot ; k) .
$$

where the eigenfunction $\psi_{j, \infty}(x ; k)$ satisfies

$$
\begin{aligned}
& -\frac{\partial^{2} \psi_{j, \infty}(x ; k)}{\partial x^{2}}+(b x-k)^{2} \psi_{j, \infty}(x ; k) \\
& \quad=b(2 j-1) \psi_{j, \infty}(x ; k), \quad\left\|\psi_{j, \infty}(\cdot ; k)\right\|_{L^{2}(\mathbb{R})}=1 .
\end{aligned}
$$

Moreover, $\psi_{j, \infty}(\cdot ; k)$ could be chosen to be real-valued. The functions $\psi_{j, \infty}, j \in \mathbb{N}$, admit an explicit description. Namely, if we put

$$
\varphi_{j}(x)=\frac{H_{j-1}(x) e^{-x^{2} / 2}}{\left(\sqrt{\pi} 2^{j-1}(j-1) !\right)^{1 / 2}}, \quad x \in \mathbb{R}, j \in \mathbb{N},
$$

where

$$
\mathrm{H}_{q}(x)=(-1)^{q} e^{x^{2}} \frac{d^{q}}{d x^{q}} e^{-x^{2}}, \quad x \in \mathbb{R}, q \in \mathbb{Z}_{+},
$$

are the Hermite polynomials (see, e.g., [1], Chapter I, eqs. (8.5) and (8.7)), then the real-valued function $\varphi_{j}$ satisfies

$$
-\varphi_{j}^{\prime \prime}(x)+x^{2} \varphi_{j}(x)=(2 j-1) \varphi_{j}(x), \quad\left\|\varphi_{j}\right\|_{L^{2}(\mathbb{R})}=1,
$$

and we have

$$
\psi_{j, \infty}(x ; k)=b^{1 / 4} \varphi_{j}\left(b^{1 / 2} x-b^{-1 / 2} k\right), \quad j \in \mathbb{N}, x \in \mathbb{R}, k \in \mathbb{R} .
$$

Put

$$
p_{j}=p_{j}(b)=\frac{b^{-j+3 / 2}}{\sqrt{\pi}(j-1) ! 2^{j-1}}, \quad j \in \mathbb{N} .
$$

Note that

$$
\psi_{j, \infty}(x ; k)=p_{j}^{1 / 2}(-k)^{j-1} e^{-\left(b^{-1 / 2} k-b^{1 / 2} x\right)^{2} / 2}(1+o(1))
$$

as $k \rightarrow \infty$, uniformly with respect to $x$ belonging to a compact subset of $\mathbb{R}$.

Theorem 2.3. Fix $j \in \mathbb{N}$. Then we have

$$
\lim _{k \rightarrow \infty}\left(\varepsilon_{j}^{+}-E_{j}(k)\right)^{-1 / 2}\left\|\pi_{j, \infty}-\pi_{j}(k)\right\|_{1}=0
$$

where $\|T\|_{1}$ denotes the trace-class norm of the operator $T$. 
We will divide the proof of the theorem into several propositions.

By analogy with (2.4) and (2.6) set

$$
\tilde{\pi}_{j}(k)=\left\langle\cdot, \tilde{\psi}_{j}(\cdot ; k)\right\rangle \tilde{\psi}_{j}(\cdot ; k), \quad k \in \mathbb{R}, \quad \tilde{\pi}_{j, \infty}=\left\langle\cdot, \tilde{\psi}_{j, \infty}\right\rangle \tilde{\psi}_{j, \infty}, \quad j \in \mathbb{N},
$$

where

$$
\tilde{\psi}_{j}(\cdot ; k)=\tau_{k}^{*} \psi_{j}(\cdot ; k), \quad \tilde{\psi}_{j, \infty}=\tau_{k}^{*} \psi_{j, \infty},
$$

the function $\psi_{j}(\cdot ; k)$ (resp., $\psi_{j, \infty}$ ) being introduced in (2.5) (resp., in (2.7)). Evidently,

$$
\begin{aligned}
& \tilde{h}(k) \tilde{\psi}_{j}(\cdot ; k)=E_{j}(k) \tilde{\psi}_{j}(\cdot ; k), \quad\left\|\tilde{\psi}_{j}(\cdot ; k)\right\|_{L^{2}(\mathbb{R})}=1, \\
& \tilde{\psi}_{j, \infty}(x)=b^{1 / 4} \varphi_{j}\left(b^{1 / 2} x\right), \quad x \in \mathbb{R},
\end{aligned}
$$

the function $\varphi_{j}$ being defined in (2.8), and

$$
\tilde{h}_{\infty} \tilde{\psi}_{j, \infty}=\varepsilon_{j}^{+} \tilde{\psi}_{j, \infty}, \quad\left\|\tilde{\psi}_{j, \infty}\right\|_{L^{2}(\mathbb{R})}=1 .
$$

Since we have

$$
\tau_{k} \tilde{\pi}_{j}(k) \tau_{k}^{*}=\pi_{j}(k), \quad \tau_{k} \tilde{\pi}_{j, \infty} \tau_{k}^{*}=\pi_{j, \infty}(k), \quad k \in \mathbb{R}, j \in \mathbb{N},
$$

relation (2.12) is equivalent to

$$
\lim _{k \rightarrow \infty}\left(\varepsilon_{j}^{+}-E_{j}(k)\right)^{-1 / 2}\left\|\tilde{\pi}_{j, \infty}-\tilde{\pi}_{j}(k)\right\|_{1}=0 .
$$

For $z \in \mathbb{C} \backslash\left(\sigma\left(\tilde{h}_{\infty}\right) \backslash\left\{\varepsilon_{j}^{+}\right\}\right)$set

$$
R_{0, j}^{\perp}(z)=\left(\tilde{h}_{\infty}-z\right)^{-1}\left(I-\tilde{\pi}_{j, \infty}\right) .
$$

Similarly, for $z \in \mathbb{C} \backslash\left(\sigma(\tilde{h}(k)) \backslash\left\{E_{j}(k)\right\}\right)$ put

$$
R_{j}^{\perp}(z)=(\tilde{h}(k)-z)^{-1}\left(I-\tilde{\pi}_{j}(k)\right) .
$$

Set

$$
U_{k}(x)=W_{+}-W(x+k / b)=\tilde{h}_{\infty}-\tilde{h}(k), \quad x \in \mathbb{R}, k \in \mathbb{R} .
$$

Proposition 2.4. We have

$$
\begin{aligned}
\tilde{\pi}_{j, \infty} & =\tilde{\pi}_{j, \infty} \tilde{\pi}_{j}(k)-\tilde{\pi}_{j, \infty} U_{k} R_{j}^{\perp}\left(\mathcal{E}_{j}^{+}\right) \\
& =\tilde{\pi}_{j}(k) \tilde{\pi}_{j, \infty}-R_{j}^{\perp}\left(\varepsilon_{j}^{+}\right) U_{k} \tilde{\pi}_{j, \infty}
\end{aligned}
$$

and

$$
\begin{aligned}
\tilde{\pi}_{j}(k) & =\tilde{\pi}_{j}(k) \tilde{\pi}_{j, \infty}+\tilde{\pi}_{j}(k) U_{k} R_{0, j}^{\perp}\left(E_{j}\right) \\
& =\tilde{\pi}_{j, \infty} \tilde{\pi}_{j}(k)+R_{0, j}^{\perp}\left(E_{j}\right) U_{k} \tilde{\pi}_{j}(k) .
\end{aligned}
$$


Proof. We have

$$
\begin{aligned}
\tilde{\pi}_{j, \infty} & =\tilde{\pi}_{j, \infty} \tilde{\pi}_{j}(k)+\tilde{\pi}_{j, \infty}\left(I-\tilde{\pi}_{j}(k)\right) \\
& =\tilde{\pi}_{j, \infty} \tilde{\pi}_{j}(k)+\tilde{\pi}_{j, \infty}\left(\tilde{h}_{\infty}-\varepsilon_{j}^{+}-U_{k}\right)\left(\tilde{h}(k)-\varepsilon_{j}^{+}\right)^{-1}\left(I-\tilde{\pi}_{j}(k)\right) .
\end{aligned}
$$

Since $\tilde{\pi}_{j, \infty}\left(\tilde{h}_{\infty}-\varepsilon_{j}^{+}\right)=0$, we obtain the first equality in (2.14). The second equality is obtained by taking the adjoint. In relations (2.15) we have only exchanged the role of $\tilde{h}(k)$ and $\tilde{h}_{\infty}$.

Set

$$
\Phi_{j}(k)=\Phi_{j}(k ; W)=\left(\int_{\mathbb{R}} U_{k}(x) \tilde{\psi}_{j, \infty}(x)^{2} d x\right)^{1 / 2}, \quad k \in \mathbb{R} .
$$

By the dominated convergence theorem we have $\lim _{k \rightarrow+\infty} \Phi_{j}(k)=0$. Note that

$$
\begin{aligned}
\Phi_{j}(k) & =\left(\operatorname{Tr} \tilde{\pi}_{j, \infty} U_{k} \tilde{\pi}_{j, \infty}\right)^{1 / 2}=\left\|\tilde{\pi}_{j, \infty} U_{k}^{1 / 2}\right\|_{1}=\left\|U_{k}^{1 / 2} \tilde{\pi}_{j, \infty}\right\|_{1} \\
& =\left\|\tilde{\pi}_{j, \infty} U_{k}^{1 / 2}\right\|=\left\|U_{k}^{1 / 2} \tilde{\pi}_{j, \infty}\right\| .
\end{aligned}
$$

Corollary 2.5. Fix $j \in \mathbb{N}$. Then we have

$$
\left\|\tilde{\pi}_{j, \infty}-\tilde{\pi}_{j}(k)\right\|_{1}=o\left(\Phi_{j}(k)\right), \quad k \rightarrow \infty .
$$

Proof. By (2.14) and (2.15) we have

$\tilde{\pi}_{j, \infty}-\tilde{\pi}_{j}(k)=-\tilde{\pi}_{j, \infty} U_{k} R_{j}^{\perp}\left(\mathcal{E}_{j}^{+}\right)-R_{0, j}^{\perp}\left(E_{j}\right) U_{k} \tilde{\pi}_{j, \infty}+R_{0, j}^{\perp}\left(E_{j}\right) U_{k}\left(\tilde{\pi}_{j, \infty}-\tilde{\pi}_{j}(k)\right)$, i.e.

$$
\left(I-R_{0, j}^{\perp}\left(E_{j}\right) U_{k}\right)\left(\tilde{\pi}_{j, \infty}-\tilde{\pi}_{j}(k)\right)=-\tilde{\pi}_{j, \infty} U_{k} R_{j}^{\perp}\left(\varepsilon_{j}^{+}\right)-R_{0, j}^{\perp}\left(E_{j}\right) U_{k} \tilde{\pi}_{j, \infty} .
$$

Since $s-\lim _{k \rightarrow \infty} U_{k}=0$ and the operator $R_{0, j}^{\perp}\left(E_{j}\right)$ is compact and uniformly bounded, we have $\lim _{k \rightarrow \infty}\left\|R_{0, j}^{\perp}\left(E_{j}\right) U_{k}\right\|=0$, so the operator $I-R_{0, j}^{\perp}\left(E_{j}\right) U_{k}$ is invertible for sufficiently great $k$, and for such $k$ we have

$$
\tilde{\pi}_{j, \infty}-\tilde{\pi}_{j}(k)=-\left(I-R_{0, j}^{\perp}\left(E_{j}\right) U_{k}\right)^{-1}\left(\tilde{\pi}_{j, \infty} U_{k} R_{j}^{\perp}\left(\varepsilon_{j}^{+}\right)+R_{0, j}^{\perp}\left(E_{j}\right) U_{k} \tilde{\pi}_{j, \infty}\right) .
$$

Therefore,

$$
\begin{aligned}
\left\|\tilde{\pi}_{j, \infty}-\tilde{\pi}_{j}(k)\right\|_{1} & \leq\left\|\left(I-R_{0, j}^{\perp}\left(E_{j}\right) U_{k}\right)^{-1}\right\|\left(\left\|U_{k}^{1 / 2} R_{j}^{\perp}\left(\varepsilon_{j}^{+}\right)\right\|\right. \\
& \left.+\left\|R_{0, j}^{\perp}\left(E_{j}\right) U_{k}^{1 / 2}\right\|\right)\left\|\tilde{\pi}_{j, \infty} U_{k}^{1 / 2}\right\|_{1} .
\end{aligned}
$$

Arguing as above, we easily find that

$$
\lim _{k \rightarrow \infty}\left\|U_{k}^{1 / 2} R_{j}^{\perp}\left(\mathcal{E}_{j}^{+}\right)\right\|=\lim _{k \rightarrow \infty}\left\|R_{0, j}^{\perp}\left(E_{j}\right) U_{k}^{1 / 2}\right\|=0 .
$$

Now the combination of (2.19), (2.20), and (2.17) implies (2.18). 
Proposition 2.6. We have

$$
\varepsilon_{j}^{+}-E_{j}(k)=\Phi_{j}(k)^{2}(1+o(1)), \quad k \rightarrow \infty .
$$

Proof. Assume $k$ large enough. Evidently,

$$
\begin{aligned}
\mathcal{E}_{j}^{+} & =\operatorname{Tr} \tilde{h}_{\infty} \tilde{\pi}_{j, \infty}=-\frac{1}{2 \pi i} \operatorname{Tr} \int_{\Gamma_{j}} \tilde{h}_{\infty}\left(\tilde{h}_{\infty}-\omega\right)^{-1} d \omega \\
& =-\frac{1}{2 \pi i} \operatorname{Tr} \int_{\Gamma_{j}} \omega\left(\tilde{h}_{\infty}-\omega\right)^{-1} d \omega
\end{aligned}
$$

where $\Gamma_{j}$ is a sufficiently small circle run over in the anticlockwise direction which contains in its interior $E_{j}(k)$ and $\varepsilon_{j}^{+}$but no other points from the spectra of $\tilde{h}(k)$ and $\tilde{h}_{\infty}$. Similarly,

$$
E_{j}(k)=-\frac{1}{2 \pi i} \operatorname{Tr} \int_{\Gamma_{j}} \omega(\tilde{h}(k)-\omega)^{-1} d \omega
$$

Therefore,

$$
\begin{aligned}
\mathcal{E}_{j}^{+}-E_{j}(k) & =-\frac{1}{2 \pi i} \operatorname{Tr} \int_{\Gamma_{j}} \omega\left(\left(\tilde{h}_{\infty}-\omega\right)^{-1}-(\tilde{h}(k)-\omega)^{-1}\right) d \omega \\
& =\frac{1}{2 \pi i} \operatorname{Tr} \int_{\Gamma_{j}} \omega\left(\tilde{h}_{\infty}-\omega\right)^{-1} U_{k}(\tilde{h}(k)-\omega)^{-1} d \omega .
\end{aligned}
$$

Applying the Cauchy theorem, we easily get

$$
\begin{aligned}
& \frac{1}{2 \pi i} \int_{\Gamma_{j}} \omega\left(\tilde{h}_{\infty}-\omega\right)^{-1} U_{k}(\tilde{h}(k)-\omega)^{-1} d \omega \\
& \quad=\tilde{\pi}_{j, \infty} U_{k} \tilde{\pi}_{j}(k)-\mathcal{E}_{j}^{+} \tilde{\pi}_{j, \infty} U_{k} R_{j}^{\perp}\left(\mathcal{E}_{j}^{+}\right)-E_{j} R_{0, j}^{\perp}\left(E_{j}\right) U_{k} \tilde{\pi}_{j}(k) .
\end{aligned}
$$

Comparing (2.22) and (2.23), and bearing in mind (2.17), we obtain

$$
\begin{aligned}
\mathcal{E}_{j}^{+}-E_{j}(k)-\Phi_{j}(k)^{2}=\operatorname{Tr} & \tilde{\pi}_{j, \infty} U_{k}\left(\tilde{\pi}_{j}(k)-\tilde{\pi}_{j, \infty}\right) \\
& -\mathcal{E}_{j}^{+} \operatorname{Tr} \tilde{\pi}_{j, \infty} U_{k} R_{j}^{\perp}\left(\varepsilon_{j}^{+}\right) \\
& -E_{j} \operatorname{Tr} R_{0, j}^{\perp}\left(E_{j}\right) U_{k} \tilde{\pi}_{j}(k) .
\end{aligned}
$$

In order to complete the proof of (2.21), it remains to show that the three terms on the r.h.s. of (2.24) are of order $o\left(\Phi_{j}(k)^{2}\right)$ as $k \rightarrow \infty$.

First, we have

$$
\begin{aligned}
\left|\operatorname{Tr} \tilde{\pi}_{j, \infty} U_{k}\left(\tilde{\pi}_{j}(k)-\tilde{\pi}_{j, \infty}\right)\right| & \leq\left\|\tilde{\pi}_{j, \infty} U_{k}^{1 / 2}\right\|\left\|U_{k}^{1 / 2}\right\|\left\|\tilde{\pi}_{j}(k)-\tilde{\pi}_{j, \infty}\right\|_{1} \\
& =o\left(\Phi_{j}(k)^{2}\right), \quad k \rightarrow \infty,
\end{aligned}
$$

by (2.17), (2.18), and the fact that $\left\|U_{k}^{1 / 2}\right\|$ is uniformly bounded with respect to $k \in \mathbb{R}$. 
Next, using the trivial identities $\tilde{\pi}_{j, \infty}=\tilde{\pi}_{j, \infty}^{2}$ and $R_{j}^{\perp}\left(\mathcal{E}_{j}^{+}\right) \tilde{\pi}_{j}(k)=0$, as well as the cyclicity of the trace, we obtain

$$
\operatorname{Tr} \tilde{\pi}_{j, \infty} U_{k} R_{j}^{\perp}\left(\varepsilon_{j}^{+}\right)=-\operatorname{Tr}\left(\tilde{\pi}_{j}(k)-\tilde{\pi}_{j, \infty}\right) \tilde{\pi}_{j, \infty} U_{k} R_{j}^{\perp}\left(\mathcal{E}_{j}^{+}\right) .
$$

Therefore, similarly to (2.25), we have

$$
\begin{aligned}
\left|\mathcal{E}_{j}^{+} \operatorname{Tr} \tilde{\pi}_{j, \infty} U_{k} R_{j}^{\perp}\left(\mathcal{E}_{j}^{+}\right)\right| & \leq\left|\mathcal{E}_{j}^{+}\right|\left\|\tilde{\pi}_{j}(k)-\tilde{\pi}_{j, \infty}\right\|_{1} \\
& \left\|\tilde{\pi}_{j, \infty} U_{k}^{1 / 2}\right\|\left\|U_{k}^{1 / 2} R_{j}^{\perp}\left(\mathcal{E}_{j}^{+}\right)\right\| \\
& =o\left(\Phi_{j}(k)^{2}\right)
\end{aligned}
$$

as $k \rightarrow \infty$. Finally, by analogy with (2.26) we have

$$
\begin{aligned}
\operatorname{Tr} R_{0, j}^{\perp}\left(E_{j}\right) U_{k} \tilde{\pi}_{j}(k)= & \operatorname{Tr} R_{0, j}^{\perp}\left(E_{j}\right) U_{k} \tilde{\pi}_{j}(k)\left(\tilde{\pi}_{j}(k)-\tilde{\pi}_{j, \infty}\right) \\
= & \operatorname{Tr} R_{0, j}^{\perp}\left(E_{j}\right) U_{k} \tilde{\pi}_{j, \infty}\left(\tilde{\pi}_{j}(k)-\tilde{\pi}_{j, \infty}\right) \\
& +\operatorname{Tr} R_{0, j}^{\perp}\left(E_{j}\right) U_{k}\left(\tilde{\pi}_{j}(k)-\tilde{\pi}_{j, \infty}\right)^{2} .
\end{aligned}
$$

Hence,

$$
\begin{aligned}
& \left|E_{j} \operatorname{Tr} R_{0, j}^{\perp}\left(E_{j}\right) U_{k} \tilde{\pi}_{j}(k)\right| \\
& \quad \leq\left|E_{j}(k)\right|\left\|R_{0, j}^{\perp}\left(E_{j}\right) U_{k}^{1 / 2}\right\|\left\|U_{k}^{1 / 2} \tilde{\pi}_{j, \infty}\right\|\left\|\tilde{\pi}_{j}(k)-\tilde{\pi}_{j, \infty}\right\|_{1} \\
& \quad+\left|E_{j}(k)\right|\left\|R_{0, j}^{\perp}\left(E_{j}\right) U_{k}\right\|\left\|\tilde{\pi}_{j}(k)-\tilde{\pi}_{j, \infty}\right\|_{1}^{2} \\
& \quad=o\left(\Phi_{j}(k)^{2}\right), \quad k \rightarrow \infty,
\end{aligned}
$$

by (2.17), (2.18), and the fact that both $\left|E_{j}(k)\right|$ and $\left\|R_{0, j}^{\perp}\left(E_{j}(k)\right) U_{k}^{1 / 2}\right\|$, as well as $\left\|R_{0, j}^{\perp}\left(E_{j}(k)\right) U_{k}\right\|$ are uniformly bounded with respect to $k \in \mathbb{R}$.

Putting together (2.24), (2.25), (2.27), and (2.28), we obtain (2.21).

Now (2.13) (and, hence, (2.12)) follows immediately from (2.18) and (2.21).

\section{Effective Hamiltonians}

Assume that $W$ is a non-decreasing function, and (1.4) holds true. As explained in the introduction, for definiteness, we will consider the case of positive perturbations, and respectively the asymptotic behavior as $\lambda \downarrow 0$ of $\mathcal{N}_{j}^{+}(\lambda), j \in \mathbb{N}, \lambda \in(0,2 b+$ $\left.W_{-}-W_{+}\right)$.

Pick $j \in \mathbb{N}, A \in[-\infty, \infty)$ and $\lambda>0$, and set

$$
\begin{aligned}
& P_{j}(A)=\int_{(A, \infty)}^{\oplus} \pi_{j}(k) d k, \\
& \mathcal{P}_{j}(A)=\mathscr{F}^{*} P_{j}(A) \mathcal{F},
\end{aligned}
$$




$$
\begin{aligned}
P_{j, \infty}(A) & =\int_{(A, \infty)}^{\oplus} \pi_{j, \infty}(k) d k, \\
T_{j}(\lambda ; A) & =\int_{(A, \infty)}^{\oplus}\left(\mathcal{E}_{j}^{+}-E_{j}(k)+\lambda\right)^{-1 / 2} \pi_{j}(k) d k, \\
T_{j, \infty}(\lambda ; A) & =\int_{(A, \infty)}^{\oplus}\left(\mathcal{E}_{j}^{+}-E_{j}(k)+\lambda\right)^{-1 / 2} \pi_{j, \infty}(k) d k .
\end{aligned}
$$

Proposition 3.1. Assume $M \in L_{0}^{\infty}\left(\mathbb{R}^{2}\right)$. Then the operator $M \mathcal{F}^{*} T_{j, \infty}(\lambda ; A)$ is compact for any $\lambda>0$ and $A \in[-\infty, \infty)$. Moreover, for any $A_{1}, A_{2} \in[-\infty, \infty)$ the operator

$$
M \mathcal{F}^{*}\left(T_{j, \infty}\left(\lambda ; A_{1}\right)-T_{j, \infty}\left(\lambda ; A_{2}\right)\right)
$$

extends to a uniformly bounded and continuous operator for $\lambda \geq 0$.

Proof. Denote by $\chi_{R}$ the characteristic function of a disk of radius $R$ centered at the origin. For $\lambda>0$ and $A \in[-\infty, \infty)$ write

$$
M \mathscr{F}^{*} T_{j, \infty}(\lambda ; A)=\chi_{R} M \mathscr{F}^{*} T_{j, \infty}(\lambda ; A)+\left(1-\chi_{R}\right) M \mathcal{F}^{*} T_{j, \infty}(\lambda ; A) .
$$

The first operator at the r.h.s of (3.2) is Hilbert-Schmidt for any $R \in(0, \infty)$, and the norm of the second one tends to zero as $R \rightarrow \infty$. Hence, the operator $M \mathscr{F}^{*} T_{j, \infty}(\lambda ; A)$ is compact. Further, the case $A_{1}=A_{2}$ in (3.1) is trivial so that we suppose $A_{1} \neq A_{2}$. Define the value for $\lambda=0$ of the operator in (3.1) as

$$
M \mathscr{F}^{*} \int_{\left(A_{-}, A_{+}\right)}^{\oplus}\left(\mathcal{E}_{j}^{+}-E_{j}(k)\right)^{-1 / 2} \pi_{j, \infty}(k) d k
$$

with $A_{-}=\min \left\{A_{1}, A_{2}\right\}$ and $A_{+}=\max \left\{A_{1}, A_{2}\right\}$. Now the uniform boundedness for $\lambda \geq 0$ of the operator in (3.1) follows from the estimates

$$
\begin{aligned}
& \left\|M \mathcal{F}^{*}\left(T_{j, \infty}\left(\lambda ; A_{1}\right)-T_{j, \infty}\left(\lambda ; A_{2}\right)\right)\right\| \\
& \quad \leq\|M\|_{L \infty\left(\mathbb{R}^{2}\right)} \sup _{k \in\left(A_{-}, A_{+}\right]}\left(\mathcal{E}_{j}^{+}-E_{j}(k)\right)^{-1 / 2}, \quad \lambda \geq 0,
\end{aligned}
$$

while the uniform continuity of this operator for $\lambda \geq 0$ follows from the estimates

$$
\begin{gathered}
\left\|M \mathcal{F}^{*}\left(\left(T_{j, \infty}\left(\lambda_{1} ; A_{1}\right)-T_{j, \infty}\left(\lambda_{1} ; A_{2}\right)\right)-\left(T_{j, \infty}\left(\lambda_{2} ; A_{1}\right)-T_{j, \infty}\left(\lambda_{2} ; A_{2}\right)\right)\right)\right\| \\
\quad \leq\left|\lambda_{1}-\lambda_{2}\right|\|M\|_{L^{\infty}\left(\mathbb{R}^{2}\right)} \sup _{k \in\left(A_{-}, A_{+}\right]}\left(\mathcal{E}_{j}^{+}-E_{j}(k)\right)^{-2}, \quad \lambda_{1}, \lambda_{2} \geq 0 .
\end{gathered}
$$

Let $s>0$ and $T=T^{*}$ be a linear compact operator acting in a given Hilbert space. ${ }^{1}$ Set

$$
n_{ \pm}(s ; T)=\operatorname{rank} \mathbb{P}_{(s, \infty)}( \pm T) ;
$$

\footnotetext{
${ }^{1}$ All Hilbert spaces in this article are supposed to be separable.
} 
thus the functions $n_{ \pm}(\cdot ; T)$ are respectively the counting functions of the positive and negative eigenvalues of the operator $T$. In the case where $T$ is compact but not necessarily self-adjoint (in particular, $T$ could act between two different Hilbert spaces), we will use also the notations

$$
n_{*}(s ; T)=n_{+}\left(s^{2} ; T^{*} T\right), \quad s>0 ;
$$

thus $n_{*}(\cdot ; T)$ is the counting function of the singular values of $T$. Of course, we have $n_{*}(s ; T)=n_{*}\left(s ; T^{*}\right)$ and hence $n_{+}\left(s ; T^{*} T\right)=n_{+}\left(s ; T T^{*}\right)$ for any $s>0$. For further references we recall here the well-known Weyl inequalities

$$
n_{+}\left(s_{1}+s_{2} ; T_{1}+T_{2}\right) \leq n_{+}\left(s_{1} ; T_{1}\right)+n_{+}\left(s_{2} ; T_{2}\right)
$$

where $s_{j}>0$, and $T_{j}, j=1,2$, are linear self-adjoint operators acting in a given Hilbert space. In the case where $T_{1}$ and $T_{2}$ are linear and compact but not necessarily self-adjoint, we recall also the Ky-Fan inequalities

$$
n_{*}\left(s_{1}+s_{2} ; T_{1}+T_{2}\right) \leq n_{*}\left(s_{1} ; T_{1}\right)+n_{*}\left(s_{2} ; T_{2}\right), \quad s_{1}, s_{2}>0 .
$$

Theorem 3.2. Assume that $V \in L_{0}^{\infty}\left(\mathbb{R}^{2} ; \mathbb{R}\right)$. Fix $j \in \mathbb{N}$ and $A \in[-\infty, \infty)$. Then for any $\varepsilon \in(0,1)$ we have

$$
\begin{aligned}
n_{+}(1 & \left.+\varepsilon ; T_{j, \infty}(\lambda ; A) \mathcal{F} V \mathscr{F}^{*} T_{j, \infty}(\lambda ; A)\right)+O(1) \\
& \leq \mathcal{N}_{j}^{+}(\lambda) \\
& \leq n_{+}\left(1-\varepsilon ; T_{j, \infty}(\lambda ; A) \mathcal{F} V \mathscr{F}^{*} T_{j, \infty}(\lambda ; A)\right)+O(1), \quad \lambda \downarrow 0 .
\end{aligned}
$$

Proof. The Birman-Schwinger principle implies

$$
\mathcal{N}_{j}^{+}(\lambda)=n_{-}\left(1 ; V^{1 / 2}\left(H_{0}-\varepsilon_{j}^{+}-\lambda\right)^{-1} V^{1 / 2}\right)+O(1), \quad \lambda \downarrow 0 .
$$

Pick $\widetilde{A} \in \mathbb{R}$. Applying the Weyl inequalities (3.3), we get

$$
\begin{aligned}
n_{+}\left(1+s ; V^{1 / 2}\left(\mathcal{E}_{j}^{+}-H_{0}+\lambda\right)^{-1} \mathcal{P}_{j, \widetilde{A}} V^{1 / 2}\right) \\
-n_{-}\left(s ; V^{1 / 2}\left(\mathcal{E}_{j}^{+}-H_{0}+\lambda\right)^{-1}\left(I-\mathcal{P}_{j, \widetilde{A}}\right) V^{1 / 2}\right) \\
\leq n_{-}\left(1 ; V^{1 / 2}\left(H_{0}-\mathcal{E}_{j}^{+}-\lambda\right)^{-1} V^{1 / 2}\right) \\
\leq n_{+}\left(1-s ; V^{1 / 2}\left(\mathcal{E}_{j}^{+}-H_{0}+\lambda\right)^{-1} \mathcal{P}_{j, \tilde{A}} V^{1 / 2}\right) \\
\quad+n_{+}\left(s ; V^{1 / 2}\left(\varepsilon_{j}^{+}-H_{0}+\lambda\right)^{-1}\left(I-\mathcal{P}_{j, \widetilde{A}}\right) V^{1 / 2}\right),
\end{aligned}
$$

for any $s \in(0,1)$. By $V \in L_{0}^{\infty}\left(\mathbb{R}^{2} ; \mathbb{R}\right)$ and the diamagnetic inequality, we easily find that

$$
n_{ \pm}\left(s ; V^{1 / 2}\left(\mathcal{E}_{j}^{+}-H_{0}+\lambda\right)^{-1}\left(I-\mathcal{P}_{j, \widetilde{A}}\right) V^{1 / 2}\right)=O(1), \quad \lambda \downarrow 0 .
$$


Further, for any $r>0$ we have

$$
\begin{aligned}
n_{+}\left(r^{2} ; V^{1 / 2}\left(\mathcal{E}_{j}^{+}-H_{0}+\lambda\right)^{-1} \mathcal{P}_{j, \tilde{A}} V^{1 / 2}\right) \\
\quad=n_{+}\left(r^{2} ; V^{1 / 2} \widetilde{F}^{*} \int_{(\widetilde{A}, \infty)}^{\oplus}\left(\mathcal{E}_{j}^{+}-E_{j}(k)+\lambda\right)^{-1} \pi_{j}(k) d k \mathcal{F}^{1 / 2}\right) \\
\quad=n_{*}\left(r ; V^{1 / 2} \mathcal{F}^{*} T_{j}(\lambda ; \widetilde{A})\right) .
\end{aligned}
$$

Applying the Ky-Fan inequalities (3.4), we obtain

$$
\begin{aligned}
& n_{*}(r(1+s)\left.; V^{1 / 2} \widetilde{F}^{*} T_{j, \infty}(\lambda ; \widetilde{A})\right) \\
&-n_{*}\left(r s ; V^{1 / 2} \widetilde{F}^{*}\left(T_{j, \infty}(\lambda ; \widetilde{A})-T_{j}(\lambda ; \widetilde{A})\right)\right) \\
& \leq n_{*}\left(r ; V^{1 / 2} \mathcal{F}^{*} T_{j}(\lambda ; \widetilde{A})\right) \\
& \leq n_{*}\left(r(1-s) ; V^{1 / 2} \mathcal{F}^{*} T_{j, \infty}(\lambda ; \widetilde{A})\right) \\
& \quad+n_{*}\left(r s ; V^{1 / 2} \widetilde{F}^{*}\left(T_{j, \infty}(\lambda ; \widetilde{A})-T_{j}(\lambda ; \widetilde{A})\right)\right) .
\end{aligned}
$$

Now note that

$$
\begin{aligned}
& \left\|V^{1 / 2} \tilde{F}^{*}\left(T_{j, \infty}(\lambda ; \tilde{A})-T_{j}(\lambda ; \tilde{A})\right)\right\| \\
& \quad \leq\|V\|_{L^{\infty}\left(\mathbb{R}^{2}\right)}^{1 / 2} \sup _{k>\widetilde{A}}\left(\mathcal{E}_{j}^{+}-E_{j}(k)\right)^{-1 / 2}\left\|\pi_{j}(k)-\pi_{j, \infty}(k)\right\|,
\end{aligned}
$$

uniformly with respect to $\lambda>0$. By (3.11) and Theorem 2.3 we find that for each $q>0$ there exists $A_{0}=A_{0}(q)$ such that $\tilde{A} \geq A_{0}(q)$ implies

$$
\left\|V^{1 / 2} \mathcal{F}^{*}\left(T_{j, \infty}(\lambda ; \widetilde{A})-T_{j}(\lambda ; \tilde{A})\right)\right\| \leq q
$$

for each $\lambda>0$. Choosing $\tilde{A} \geq A_{0}(r s)$ in (3.10) we find then that

$$
n_{*}\left(r s ; V^{1 / 2} \mathcal{F}^{*}\left(T_{j, \infty}(\lambda ; \widetilde{A})-T_{j}(\lambda ; \widetilde{A})\right)\right)=0
$$

for each $\lambda>0$. Next, the Ky-Fan inequalities (3.4) imply that for any $\lambda>0, r>0$, $s \in(0,1)$ and $A, \tilde{A}$, we have

$$
\begin{aligned}
& n_{*}\left(r(1+s) ; V^{1 / 2} \widetilde{F}^{*} T_{j, \infty}(\lambda ; A)\right) \\
& -n_{*}\left(r s ; V^{1 / 2} \widetilde{F}^{*}\left(T_{j, \infty}(\lambda ; A)-T_{j, \infty}(\lambda ; \widetilde{A})\right)\right) \\
& \leq n_{*}\left(r ; V^{1 / 2} \mathcal{F}^{*} T_{j, \infty}(\lambda ; \widetilde{A})\right) \\
& \leq n_{*}\left(r(1-s) ; V^{1 / 2} \mathcal{F}^{*} T_{j, \infty}(\lambda ; A)\right) \\
& \quad+n_{*}\left(r s ; V^{1 / 2} \widetilde{F}^{*}\left(T_{j, \infty}(\lambda ; A)-T_{j, \infty}(\lambda ; \widetilde{A})\right)\right) .
\end{aligned}
$$

By Proposition 3.1 we have

$$
n_{*}\left(r ; V^{1 / 2} \mathcal{F}^{*}\left(T_{j, \infty}(\lambda ; A)-T_{j, \infty}(\lambda ; \tilde{A})\right)\right)=O(1), \quad \lambda \downarrow 0,
$$


for any fixed $r>0$. Finally we note that

$$
n_{*}\left(r ; V^{1 / 2} \mathscr{F}^{*} T_{j, \infty}(\lambda ; A)\right)=n_{+}\left(r^{2} ; T_{j, \infty}(\lambda ; A) \mathcal{F} V \mathscr{F}^{*} T_{j, \infty}(\lambda ; A)\right)
$$

for each $r>0, \lambda>0$. Putting together (3.6)-(3.10), and (3.12)-(3.15), we obtain (3.5).

Note that the operator $T_{j, \infty}(\lambda ; A) \mathcal{F} V \mathscr{F}^{*} T_{j, \infty}(\lambda ; A)$ regarded as an operator on the Hilbert space $P_{j, \infty}(A) L^{2}\left(\mathbb{R}^{2}\right)$, is unitarily equivalent to $S_{j}(\lambda ; A)^{*} S_{j}(\lambda ; A)$, where $S_{j}(\lambda ; A): L^{2}(A, \infty) \rightarrow L^{2}\left(\mathbb{R}^{2}\right)$ is an operator with integral kernel

$$
(2 \pi)^{-1 / 2} V(x, y)^{1 / 2} e^{i k y} \psi_{j, \infty}(x ; k)\left(\varepsilon_{j}^{+}-E_{j}(k)+\lambda\right)^{-1 / 2},
$$

for $k \in \mathbb{R},(x, y) \in \mathbb{R}^{2}$, and $\lambda>0$, the function $\psi_{j, \infty}$ being defined in (2.9). Therefore,

$$
n_{+}\left(r ; T_{j, \infty}(\lambda ; A) \mathcal{F} V \mathscr{F}^{*} T_{j, \infty}(\lambda ; A)\right)=n_{+}\left(r ; S_{j}(\lambda ; A)^{*} S_{j}(\lambda ; A)\right),
$$

for $\quad r>0$ and $\lambda>0$.

For $(x, \xi) \in T^{*} \mathbb{R}=\mathbb{R}^{2}$ and $j \in \mathbb{N}$ set

$$
\Psi_{x, \xi ; j}(k)=b^{-1 / 2} e^{-i k \xi} \psi_{j, \infty}(x ; k), \quad k \in \mathbb{R} .
$$

Note that for each $j \in \mathbb{N}$ the system $\left\{\Psi_{x, \xi ; j}\right\}(x, \xi) \in T^{*} \mathbb{R}$ is overcomplete with respect to the measure $\frac{b}{2 \pi} d x d \xi$, i.e. for each $f \in L^{2}(\mathbb{R})$ we have

$$
\frac{b}{2 \pi} \int_{T^{*} \mathbb{R}}\left|\left\langle f, \Psi_{x, \xi ; j}\right\rangle\right|^{2} d x d \xi=\int_{\mathbb{R}}|f(k)|^{2} d k
$$

(see [1] or [28], Section 24).

For $(x, \xi) \in T^{*} \mathbb{R}$ and $j \in \mathbb{N}$ set $P_{x, \xi ; j}=\left\langle\cdot, \Psi_{x, \xi ; j}\right\rangle \Psi_{x, \xi ; j}$, and introduce the operator

$$
\mathcal{V}_{j}=\frac{b}{2 \pi} \int_{T^{*} \mathbb{R}} V(x, \xi) P_{x, \xi ; j} d x d \xi,
$$

where the integral is understood in the weak sense. Then $\mathcal{V}_{j}$ can be interpreted as a $\Psi D O$ with contravariant (generalized anti-Wick) symbol $V$, see [1]. Moreover, we have

$$
S_{j}(\lambda ;-\infty)^{*} S_{j}(\lambda ;-\infty)=\left(\mathcal{E}_{j}^{+}-E_{j}+\lambda\right)^{-1 / 2} \mathcal{V}_{j}\left(\mathcal{E}_{j}^{+}-E_{j}+\lambda\right)^{-1 / 2}
$$

Bearing in mind (3.17) and (3.18), and applying the Birman-Schwinger principle, we find that (3.5) with $A=-\infty$ and $\varepsilon \in(0,1)$ can be re-written as

$$
\begin{aligned}
\operatorname{rank} & \mathbb{P}_{\left(\mathcal{E}_{j}^{+}+\lambda, \infty\right)}\left(E_{j}+(1+\varepsilon)^{-1} \mathcal{V}_{j}\right)+O(1) \\
& \leq \mathcal{N}_{j}^{+}(\lambda) \\
& \leq \operatorname{rank} \mathbb{P}_{\left(\mathcal{E}_{j}^{+}+\lambda, \infty\right)}\left(E_{j}+(1-\varepsilon)^{-1} \mathcal{V}_{j}\right)+O(1), \quad \lambda \downarrow 0 .
\end{aligned}
$$

Thus, the operator $E_{j}+\mathcal{V}_{j}$ could be interpreted as the effective Hamiltonian which governs the asymptotics of $\mathcal{N}_{j}^{+}(\lambda)$ as $\lambda \downarrow 0$, the multiplier by $E_{j}$ being its "kinetic" part, and the $\Psi D O \mathcal{V}_{j}$ being its "potential" part. 


\section{Sufficient condition for the boundedness of $\mathcal{N}_{j}^{+}(\lambda)$}

Assume that $W$ is a bounded non-decreasing function with $W_{-}<W_{+}$. Set

$$
x^{+}=\inf \left\{x \in \mathbb{R} \mid W(x)=W_{+}\right\} .
$$

By the assumption $W_{-}<W_{+}$, we have $x^{+}>-\infty$. Set

$$
\begin{gathered}
X=\{x \in \mathbb{R} \mid \text { there exists } y \in \mathbb{R} \text { such that }(x, y) \in \operatorname{ess} \operatorname{supp} V\}, \\
X^{-}=\inf \mathcal{X}, \quad X^{+}=\sup \mathcal{X} .
\end{gathered}
$$

Theorem 4.1. Let $W$ be a bounded and non-decreasing function with $W_{-}<W_{+}$ and $x^{+} \leq \infty$. Assume that $V \in L_{0}^{\infty}\left(\mathbb{R}^{2}\right), V \geq 0,-\infty<X^{-}<X^{+}<\infty$. Suppose in addition that ess $\sup _{x \in \mathbb{R}} \int_{\mathbb{R}} V(x, y) d y<\infty$, and

$$
X^{+}<x^{+} \text {. }
$$

Then we have

$$
\mathcal{N}_{j}^{+}(\lambda)=O(1), \quad \lambda \downarrow 0, j \in \mathbb{N} .
$$

In order to prove Theorem 4.1 we need some information on the asymptotic behaviour as $k \rightarrow \infty$ of the function $\Phi_{j}(k)$ defined in (2.16), which by Proposition 2.6 determines the asymptotics of $\varepsilon_{j}-E_{j}(k)$. Let $w_{-}, w_{+} \in \mathbb{R}, w_{-}<w_{+}, x_{0} \in \mathbb{R}$. Put

$$
\mathbf{w}(x)= \begin{cases}w_{+} & \text {if } x \geq x_{0}, \\ w_{-} & \text {if } x<x_{0} .\end{cases}
$$

Proposition 4.2. Assume that $w_{-}<w_{+}$. Then we have

$$
\Phi_{j}(k ; \mathbf{w})^{2}=\frac{\left(w_{+}-w_{-}\right)}{2} p_{j} k^{2 j-3} e^{-\left(b^{-1 / 2} k-b^{1 / 2} x_{0}\right)^{2}}(1+o(1)), \quad k \rightarrow \infty,
$$

the number $p_{j}$ being defined in (2.10).

We omit the simple proof of the proposition, based on the standard Laplace method for approximate evaluation of integrals depending on a large parameter.

Remark. Comparing (2.21) and (4.5), we find that under the assumptions of Proposition 4.2, we have

$$
\begin{aligned}
& E_{j}(k ; b, \mathbf{w}) \\
& \quad=\varepsilon_{j}^{+}-\frac{\left(w_{+}-w_{-}\right)}{2} p_{j} k^{2 j-3} e^{-\left(b^{-1 / 2} k-b^{1 / 2} x_{0}\right)^{2}}(1+o(1)), \quad k \rightarrow \infty .
\end{aligned}
$$

Thus, in spite of the fact that the band functions $E_{j}(k ; b, W), k \in \mathbb{R}, j \in \mathbb{N}$, imitate in many aspects the behaviour of the edge potential $W$ (see, e.g., Proposition 2.1), 
asymptotic relation (4.6) reveals an important difference: the function $\mathbf{w}$ is equal to its maximal value $w_{+}$on the interval $\left[x_{0}, \infty\right)$, while the band functions $E_{j}(k ; b, \mathbf{w}), j \in$ $\mathbb{N}$, being analytic increasing functions, never reach their suprema $\mathcal{E}_{j}^{+}$. This purely quantum effect related to the uncertainty principle, explains many of the asymptotic results obtained in the sequel.

Proof of Theorem 4.1. By the upper bound in (3.5), and (3.17), it suffices to show that

$$
n_{*}\left(r ; S_{j}(\lambda ; A)\right)=O(1), \quad \lambda \downarrow 0,
$$

for any fixed $r>0$ and $A \in[-\infty, \infty)$. We have

$$
n_{*}\left(r ; S_{j}(\lambda ; A)\right) \leq r^{-2} \operatorname{Tr} S_{j}(\lambda ; A)^{*} S_{j}(\lambda ; A)=\frac{1}{2 \pi r^{2}} \ell_{0}(\lambda)
$$

where

$$
\jmath_{0}(\lambda)=\int_{A}^{\infty} \int_{\mathbb{R}^{2}}\left(\mathcal{E}_{j}^{+}-E_{j}(k ; b, W)+\lambda\right)^{-1} \psi_{j, \infty}(x ; k)^{2} V(x, y) d x d y d k .
$$

Now pick $\tilde{x} \in\left(X^{+}, x^{+}\right)$which is possible due to (4.2), and set

$$
\tilde{W}(x)= \begin{cases}W_{+} & \text {if } x \geq \tilde{x} \\ W(\tilde{x}) & \text { if } x<\tilde{x} .\end{cases}
$$

Since $W(x) \leq \tilde{W}(x), x \in \mathbb{R}$, the mini-max principle implies

$$
\begin{aligned}
\left(\mathcal{E}_{j}^{+}\right. & \left.-E_{j}(k ; b, W)+\lambda\right)^{-1} \\
& \leq\left(\mathcal{E}_{j}^{+}-E_{j}(k ; b, \tilde{W})+\lambda\right)^{-1}, \quad k \in \mathbb{R}, \quad j \in \mathbb{N}, \quad \lambda>0 .
\end{aligned}
$$

Therefore,

$$
d_{0}(\lambda) \leq\left(\text { ess } \sup _{x \in \mathbb{R}} \int_{\mathbb{R}} V(x, y) d y\right) d_{1}(\lambda)
$$

where

$$
d_{1}(\lambda)=\int_{A}^{\infty} \int_{X^{-}}^{X^{+}}\left(\mathcal{E}_{j}^{+}-E_{j}(k ; b, \tilde{W})+\lambda\right)^{-1} \psi_{j, \infty}(x ; k)^{2} d x d k .
$$

Taking into account (2.11), (2.21), and (4.5), and bearing in mind that the interval $\left[X^{-}, X^{+}\right]$is compact, we find that for sufficiently large $A>0$ and any $\lambda \geq 0$ we have

$$
\begin{aligned}
d_{1}(\lambda) & \leq 4\left(W_{+}-W(\tilde{x})\right)^{-1} \max _{x \in\left[X^{-}, X^{+}\right]} e^{-b\left(x^{2}-\tilde{x}^{2}\right)} \int_{A}^{\infty} \int_{X^{-}}^{X^{+}} k e^{-2 k(\tilde{x}-x)} d x d k \\
& \leq 2\left(W_{+}-W(\tilde{x})\right)^{-1} \max _{x \in\left[X^{-}, X^{+}\right]} e^{-b\left(x^{2}-\tilde{x}^{2}\right)} \int_{A}^{\infty} e^{-2 k\left(\tilde{x}-X^{+}\right)} d k<\infty,
\end{aligned}
$$

due to $\tilde{x}>X^{+}$. Putting together (4.8) - (4.11), we obtain (4.7), and hence (4.3). 


\section{Reduction to operators in holomorphic spaces}

In the sequel we assume that there exist bounded domains $\Omega_{ \pm} \subset \mathbb{R}^{2}$ with Lipschitz boundaries, and constants $c_{0}^{ \pm}>0$ such that

$$
c_{0}^{-} \chi_{\Omega_{-}}(x, y) \leq V(x, y) \leq c_{0}^{+} \chi_{\Omega_{+}}(x, y), \quad(x, y) \in \mathbb{R}^{2},
$$

where $\chi_{\Omega_{ \pm}}$denotes the characteristic function of the domain $\Omega_{ \pm}$. Next, for $\delta \in$ $(0,1 / 2)$ introduce the intervals

$$
I_{-}=I_{-}(\delta)=(\delta, 1-\delta), \quad I_{+}=I_{+}(\delta)=(0,1+\delta) .
$$

In what follows we will assume that the infimum $x^{+}$defined in (4.1) satisfies $x^{+}<\infty$, because in the case $x^{+}=\infty$ Theorem 4.1 implies $\mathcal{N}_{j}^{+}(\lambda)=O(1)$ as $\lambda \downarrow 0$. Since the operator $H_{0}$ is invariant under magnetic translations, we will assume that $x^{+}=0$ without any loss of generality.

Let $\delta \in(0,1 / 2)$ and $m>0$. Define the operator $\Gamma_{\delta}^{-}(m): L^{2}\left(I_{-}\right) \rightarrow L^{2}\left(\Omega_{-}\right)$as the operator with integral kernel

$$
\pi^{-1 / 2} m e^{-b x^{2} / 2} e^{m(x+i y) k} k^{1 / 2}, \quad k \in I_{-},(x, y) \in \Omega_{-},
$$

and the operator $\Gamma_{\delta}^{+}(m): L^{2}\left(I_{+}\right) \rightarrow L^{2}\left(\Omega_{+}\right)$as the operator with integral kernel

$$
\pi^{-1 / 2} m e^{-b x^{2} / 2} e^{m(x+i y+\delta) k} k^{1 / 2}, \quad k \in I_{+},(x, y) \in \Omega_{+} .
$$

Remark. Introduce the set

$$
\mathscr{B}\left(\Omega_{ \pm}\right)=\left\{u \in L^{2}\left(\Omega_{ \pm}\right) \mid u \text { is analytic in } \Omega_{ \pm}\right\}
$$

considered as a subspace of the Hilbert space $L^{2}\left(\Omega_{ \pm} ; e^{-b x^{2}} d x d y\right)$. Note that as a functional set $\mathcal{B}\left(\Omega_{ \pm}\right)$coincides with the Bergman space over $\Omega_{ \pm}$(see, e.g., [9], Subsection 3.1). Then, up to unitary equivalence, the operators $\Gamma_{\delta}^{ \pm}(m) \operatorname{map} L^{2}\left(I_{ \pm}\right)$ into $\mathcal{B}\left(\Omega_{ \pm}\right)$.

Theorem 5.1. Suppose that $W$ is a bounded non-decreasing function with $W_{-}<W_{+}$. Assume that $V \in L_{0}^{\infty}\left(\mathbb{R}^{2} ; \mathbb{R}\right)$ satisfies (5.1). Then we have

$$
n_{*}\left(r ; S_{j}(\lambda ; A)\right) \geq n_{*}\left(r(1+\varepsilon) \sqrt{\left(W_{+}-W_{-}\right) / c_{0}^{-}} ; \Gamma_{\delta}^{-}(\sqrt{b|\ln \lambda|})\right)+O(1),
$$

and

$$
\begin{aligned}
& n_{*}\left(r ; S_{j}(\lambda ; A)\right) \\
& \quad \leq n_{*}\left(r(1-\varepsilon) \sqrt{\left(W_{+}-W(-\delta)\right) / c_{0}^{+}} e^{-b \delta^{2} / 2} ; \Gamma_{\delta}^{+}(\sqrt{b|\ln \lambda|})\right)+O(1),
\end{aligned}
$$

as $\lambda \downarrow 0$, for all $j \in \mathbb{N}, A>0, \varepsilon \in(0,1), \delta \in(0,1 / 2)$ and $r>0$. 
We will divide the proof of Theorem 5.1 into two propositions.

For the proof of the first one we need the following auxiliary result. Define the operators $F_{l}, l=0,1$. by

$$
\left(F_{l} v\right)(z)=\int_{\mathbb{R}} e^{z k} k^{l} v(k) d k, \quad v \in C_{0}^{\infty}(\mathbb{R}), z \in \mathbb{C} .
$$

Note that $F_{l} v$ are entire functions in $\mathbb{C}$, and $\left(F_{1} v\right)(z)=\frac{\partial\left(F_{0} v\right)}{\partial z}(z)$. Moreover, the operators $F_{l}$ can be extended as continuous operators from $\mathscr{D}_{\text {comp }}^{\prime}(\mathbb{R})$, the subclass of compactly supported distributions from the class $\mathscr{D}^{\prime}(\mathbb{R})$, dual to $C_{0}^{\infty}(\mathbb{R})$, into the space of functions entire in $\mathbb{C}$. Set

$$
f_{l}^{ \pm}[v]=\int_{\Omega_{ \pm}} e^{-b x^{2}}\left|\left(F_{l} v\right)(x+i y)\right|^{2} d x d y, \quad v \in C_{0}^{\infty}(A, \infty), l=0,1 .
$$

Denote by $D\left[f_{1}^{ \pm}\right]$the closure of $C_{0}^{\infty}(A, \infty)$ in the norm generated by the quadratic form $f_{1}^{ \pm}$.

Lemma 5.2. The quadratic form $f_{0}^{ \pm}$is closable in $D\left[f_{1}^{ \pm}\right]$, and the operator $\mathbb{F}^{ \pm}$ generated by its closure, is compact in $D\left[f_{1}^{ \pm}\right]$.

Proof. Consider $D\left[f_{1}^{ \pm}+f_{0}^{ \pm}\right]$, the closure of $C_{0}^{\infty}(A, \infty)$ in the norm generated by the quadratic form $f_{1}^{ \pm}+f_{0}^{ \pm}$. The quadratic form $f_{0}^{ \pm}$is bounded, and hence closable in $D\left[f_{1}^{ \pm}+f_{0}^{ \pm}\right]$. Denote by $\widetilde{\mathbb{F}}^{ \pm}$the operator generated by its closure in $D\left[f_{1}^{ \pm}+f_{0}^{ \pm}\right]$. For $v \in C_{0}^{\infty}(A, \infty)$ set

$$
w(x, y)=\left(F_{0} v\right)(x+i y), \quad x+i y \in \mathbb{C} .
$$

Then we have

$$
f_{0}^{ \pm}[v]=\int_{\Omega_{ \pm}} e^{-b x^{2}}|w|^{2} d x d y, \quad f_{1}^{ \pm}[v]=2 \int_{\Omega_{ \pm}} e^{-b x^{2}}|\nabla w|^{2} d x d y .
$$

Since the $\Omega_{ \pm}$is a bounded domain with a Lipschitz boundary, the Sobolev space $\mathrm{H}^{1}\left(\Omega_{ \pm}\right)$is compactly embedded in $L^{2}\left(\Omega_{ \pm}\right)$. Hence, (5.5) implies that $\widetilde{\mathbb{F}}^{ \pm}$is compact.

Let us now check that $\left\|\widetilde{\mathbb{F}}^{ \pm}\right\|<1$. Evidently, $\left\|\widetilde{\mathbb{F}}^{ \pm}\right\| \leq 1$. Assume $\left\|\widetilde{\mathbb{F}}^{ \pm}\right\|=1$. Since $\widetilde{\mathbb{F}}^{ \pm}$is compact, this means that there exists $0 \neq v^{ \pm} \in D\left[f_{1}^{ \pm}+f_{0}^{ \pm}\right]$such that $f_{1}^{ \pm}[v]=0$. Let $\left\{v_{n}^{ \pm}\right\}_{n \in \mathbb{N}}$ be a sequence of functions $v_{n}^{ \pm} \in C_{0}^{\infty}(A, \infty) \subset C_{0}^{\infty}(\mathbb{R})$ converging to $v^{ \pm}$in $D\left[f_{1}^{ \pm}+f_{0}^{ \pm}\right]$. Set $w_{n}^{ \pm}(z)=\left(F_{0} v_{n}^{ \pm}\right)(z)$. Evidently, for any $n \in \mathbb{N}$ we have $w_{n}^{ \pm} \in \mathscr{B}\left(\Omega_{ \pm}\right)$(see (5.2)). Since $\mathcal{B}\left(\Omega_{ \pm}\right)$is complete, there exists $w^{ \pm} \in \mathscr{B}\left(\Omega_{ \pm}\right)$such that $\lim _{n \rightarrow \infty}\left\|w_{n}^{ \pm}-w^{ \pm}\right\|_{\mathcal{B}\left(\Omega_{ \pm}\right)}=0$. Since $\left(F_{1} v_{n}^{ \pm}\right)(z)=\frac{\partial w_{n}^{ \pm}}{\partial z}$, it is not difficult to check that $f_{1}^{ \pm}\left[v^{ \pm}\right]=0$ implies that $w^{ \pm}$is constant in $\Omega_{ \pm}$(see, e.g., [9], Theorem 2, Exercise 1), and hence $w^{ \pm}$admits a unique analytic extension as a constant to $\mathbb{C}$. Then the distributional Paley-Wiener theorem (see, e.g., [11], 
Theorem 1.7.7) combined with [24], Theorem V.11, implies that $v^{ \pm}$is proportional to the Dirac $\delta$-function supported at $k=0$. Since $\operatorname{supp} v^{ \pm} \subset[A, \infty)$ and $A>0$ we conclude that $v^{ \pm}=0$ as an element of $\mathscr{D}^{\prime}(\mathbb{R})$, and hence $f_{1}^{ \pm}\left[v^{ \pm}\right]+f_{0}^{ \pm}\left[v^{ \pm}\right]=0$, which contradicts with the hypothesis that $v^{ \pm} \neq 0$ as an element of $D\left[f_{1}^{ \pm}+f_{0}^{ \pm}\right]$. Therefore, $\left\|\widetilde{\mathbb{F}}^{ \pm}\right\|<1$, and the quadratic form $f_{0}^{ \pm}$is bounded, and hence closable in $D\left[f_{1}^{ \pm}\right]$. Finally, the operator $\mathbb{F}^{ \pm}$generated by its closure is unitarily equivalent to $\left(I-\widetilde{\mathbb{F}}^{ \pm}\right)^{-1} \widetilde{\mathbb{F}}^{ \pm}$and therefore is compact in $D\left[f_{1}^{ \pm}\right]$.

Define the non-decreasing functions

$$
W_{0}^{-}(x)= \begin{cases}W_{+} & \text {if } x>0, \\ W_{-} & \text {if } x \leq 0\end{cases}
$$

and

$$
W_{0}^{+}(x)=W_{0}^{+}(x ; \delta)=\left\{\begin{array}{ll}
W_{+} & \text {if } x \geq-\delta, \\
W(-\delta) & \text { if } x<-\delta,
\end{array} \quad \delta>0 .\right.
$$

Since $x^{+}=0$ and $\delta>0$ we have

$$
W_{0}^{-}(x) \leq W(x) \leq W_{0}^{+}(x ; \delta), \quad x \in \mathbb{R} .
$$

Set

$$
\omega_{ \pm}=\left\{x \in \mathbb{R} \mid \text { there exists } y \in \mathbb{R} \text { such that }(x, y) \in \Omega_{ \pm}\right\} .
$$

Let $\lambda>0, A \in[-\infty, \infty)$. Fix $j \in \mathbb{N}$. Define $Q_{j}^{ \pm}(\lambda ; A): L^{2}(A, \infty) \rightarrow L^{2}\left(\Omega_{ \pm}\right)$as the operator with integral kernel

$$
\left(\frac{p_{j}}{2 \pi}\right)^{1 / 2} e^{i k y} e^{-\left(b^{1 / 2} x-b^{-1 / 2} k\right)^{2} / 2}\left(\mathcal{E}_{j}^{+}-E_{j}\left(k ; W_{0}^{ \pm}\right)+\lambda\right)^{-1 / 2}(-k)^{j-1},
$$

with $k \in(A, \infty),(x, y) \in \Omega_{ \pm}$, the number $p_{j}$ being defined in (2.10).

Proposition 5.3. Assume that $W$ and $V$ satisfy the assumptions of Theorem 5.1. Then for every $A>0, r>0$, and $\varepsilon \in(0,1)$, we have

$$
\begin{aligned}
& n_{*}\left(r(1+\varepsilon) ; \sqrt{c_{0}^{-}} Q_{j}^{-}(\lambda ; A)\right)+O(1) \\
& \quad \leq n_{*}\left(r ; S_{j}(\lambda ; A)\right) \\
& \quad \leq n_{*}\left(r(1-\varepsilon) ; \sqrt{c_{0}^{+}} Q_{j}^{+}(\lambda ; A)\right)+O(1),
\end{aligned}
$$

as $\lambda \downarrow 0$, where $S_{j}(\lambda ; A)$ is the operator defined by (3.16), $Q_{j}^{ \pm}(\lambda ; A)$ are the operators defined by (5.8), and $c_{0}^{ \pm}$are the constants occurring in (5.1).

Proof. Inequalities (5.1) and (5.6), combined with the mini-max principle, imply the estimates

$$
n_{*}\left(r ; \sqrt{c_{0}^{-}} \tilde{S}_{j}^{-}(\lambda ; A)\right) \leq n_{*}\left(r ; S_{j}(\lambda ; A)\right) \leq n_{*}\left(r ; \sqrt{c_{0}^{+}} \tilde{S}_{j}^{+}(\lambda ; A)\right)
$$


where $\widetilde{S}_{j}^{ \pm}(\lambda ; A): L^{2}(A, \infty) \rightarrow L^{2}\left(\Omega_{ \pm}\right)$is the operator with integral kernel

$$
(2 \pi)^{-1 / 2} e^{i k y} \psi_{j, \infty}(x ; k)\left(\mathcal{E}_{j}^{+}-E_{j}\left(k ; W_{0}^{ \pm}\right)+\lambda\right)^{-1 / 2}, \quad k \in \mathbb{R},(x, y) \in \Omega_{ \pm} .
$$

In the case $j=1$ inequality (5.10) yields immediately (5.9) since in this case we have $\widetilde{S}_{1}^{ \pm}(\lambda ; A)=Q_{1}^{ \pm}(\lambda ; A)$. Assume $j \geq 2$. Then we have

$$
\psi_{j, \infty}(x ; k)=p_{j}^{1 / 2} \sum_{l=0}^{j-1} P_{l, j}(x)(-k)^{j-1-l} e^{-\left(b^{-1 / 2} k-b^{1 / 2} x\right)^{2} / 2},
$$

for $x \in \mathbb{R}$, and $k \in \mathbb{R}$, where $P_{l, j}$ is a polynomial of degree less than or equal to $l$, and $P_{0, j}=1$. Therefore,

$$
\widetilde{S}_{j}^{ \pm}(\lambda ; A)=\sum_{l=0}^{j-1} P_{l, j} Q_{j}^{ \pm}(\lambda ; A) B^{l}
$$

where the operator $B: L^{2}(A, \infty) \rightarrow L^{2}(A, \infty)$ with $A>0$ is defined by

$$
(B u)(k)=k^{-1} u(k), \quad k \in(A, \infty), u \in L^{2}(A, \infty) .
$$

Further, for each $u \in L^{2}(A, \infty)$ and $\eta \in(0,1)$, we have

$$
\begin{aligned}
\left\|\tilde{S}_{j}^{-}(\lambda ; A) u\right\|_{L^{2}\left(\Omega_{-}\right)}^{2} & =\int_{\Omega_{-}}\left|\sum_{l=0}^{j-1} P_{l, j}(x)\left(Q_{j}^{-}(\lambda, A) B^{l} u\right)(x, y)\right|^{2} d x d y \\
& \geq(1-\eta) \int_{\Omega_{-}}\left|P_{0, j}(x)^{2}\left(Q_{j}^{-}(\lambda, A) B^{l} u\right)(x, y)\right|^{2} d x d y \\
& -\left(\eta^{-1}-1\right) \int_{\Omega_{-}}\left|\sum_{l=1}^{j-1} P_{l, j}(x)\left(Q_{j}^{-}(\lambda, A) B^{l} u\right)(x, y)\right|^{2} d x d y \\
& \geq(1-\eta) \int_{\Omega_{-}}\left|P_{0, j}(x)^{2}\left(Q_{j}^{-}(\lambda, A) B^{l} u\right)(x, y)\right|^{2} d x d y \\
& -\left(\eta^{-1}-1\right)(j-1) c_{2}^{-} \sum_{l=1}^{j-1} \int_{\Omega_{-}}\left|\left(Q_{j}^{-}(\lambda, A) B^{l} u\right)(x, y)\right|^{2} d x d y,
\end{aligned}
$$

with $c_{2}^{-}=\max _{l=1, \ldots, j-1} \sup _{x \in \omega_{-}} P_{l, j}(x)^{2}$, the set $\omega_{-}$being defined in (5.7). Therefore,

$$
\begin{aligned}
& \tilde{S}_{j}^{-}(\lambda ; A)^{*} \tilde{S}_{j}^{-}(\lambda ; A) \\
& \quad \geq(1-\eta) Q_{j}^{-}(\lambda ; A)^{*} Q_{j}^{-}(\lambda ; A) \\
& \quad-(j-1)\left(\eta^{-1}-1\right) c_{2}^{-} \sum_{l=1}^{j-1} B^{l} Q_{j}^{-}(\lambda ; A)^{*} Q_{j}^{-}(\lambda ; A) B^{l} .
\end{aligned}
$$


Similarly,

$$
\begin{aligned}
& \tilde{S}_{j}^{+}(\lambda ; A)^{*} \widetilde{S}_{j}^{+}(\lambda ; A) \\
& \leq(1+\eta) Q_{j}^{+}(\lambda ; A)^{*} Q_{j}^{+}(\lambda ; A) \\
& \quad+(j-1)\left(\eta^{-1}+1\right) c_{2}^{+} \sum_{l=1}^{j-1} B^{l} Q_{j}^{+}(\lambda ; A)^{*} Q_{j}^{+}(\lambda ; A) B^{l},
\end{aligned}
$$

with $\eta>0$, and $c_{2}^{+}=\max _{l=1, \ldots, j-1} \sup _{x \in \omega_{+}} P_{l, j}(x)^{2}$. Let us consider now the quadratic forms

$$
\begin{aligned}
a_{l}^{ \pm}[u] & =a_{l}^{ \pm}[u ; \lambda, j] \\
& =\frac{2 \pi}{p_{j}}\left\|Q_{j}^{ \pm}(\lambda ; A) B^{l} u\right\|_{L^{2}\left(\Omega_{ \pm}\right)}^{2} \\
& =\int_{\Omega_{ \pm}} e^{-b x^{2}} \mid \int_{A}^{\infty} e^{k(x+i y)} e^{-b^{-1} k^{2} / 2}\left(\varepsilon_{j}^{+}-E_{j}\left(k ; W_{0}^{ \pm}\right)+\lambda\right)^{-1 / 2} \\
& \left.(-k)^{j-l-1} u(k) d k\right|^{2} d x d y
\end{aligned}
$$

with $u \in C_{0}^{\infty}(A, \infty), \lambda>0, j \geq 2, l=0, \ldots, j-2$. Evidently, $a_{l}^{ \pm}[u] \geq 0$, and $a_{l}^{ \pm}[u]=0$ implies $u=0$. Denote by $D\left[a_{l}^{ \pm}\right], l=0, \ldots, j-2$, the completion of $C_{0}^{\infty}(A, \infty)$ in the norm generated by $a_{l}^{ \pm}$.

Further, for $j \geq 2, l=0, \ldots, j-2$, and $\lambda>0$, define the operator $U_{j, l, \lambda}$ by

$$
\left(u_{j, l, \lambda}^{ \pm} u\right)(k)=e^{-b^{-1} k^{2} / 2}\left(\varepsilon_{j}^{+}-E_{j}\left(k ; W_{0}^{ \pm}\right)+\lambda\right)^{-1 / 2} k^{j-l-2} u(k), \quad k \in(A, \infty) .
$$

Note that the mapping $U_{j, l, \lambda}^{ \pm}: C_{0}^{\infty}(A, \infty) \rightarrow C_{0}^{\infty}(A, \infty)$ is bijective, and we have

$$
a_{l}^{ \pm}[u]=f_{1}^{ \pm}\left[u_{j, l, \lambda}^{ \pm} u\right], \quad a_{l+1}^{ \pm}[u]=f_{0}^{ \pm}\left[u_{j, l, \lambda}^{ \pm} u\right],
$$

for $u \in C_{0}^{\infty}(A, \infty)$ and $l=0, \ldots, j-2$. It follows from Lemma 5.2 that the quadratic form $a_{l+1}^{ \pm}$is closable in $D\left[a_{l}^{ \pm}\right], l=0, \ldots, j-2$. Denote by $\mathbb{A}_{l}^{ \pm}$the operator generated in $D\left[a_{l}^{ \pm}\right]$by the closure of the quadratic form $a_{l+1}^{ \pm}$. Since $\mathbb{A}_{l}^{ \pm}=$ $\left(U_{j, l, \lambda}^{ \pm}\right)^{-1} \mathbb{F}^{ \pm} U_{j, l, \lambda}^{ \pm}$, i.e. the operator $\mathbb{A}_{l}^{ \pm}$is unitarily equivalent to $\mathbb{F}^{ \pm}$, and $\mathbb{F}^{ \pm}$does not depend on $\lambda$, we find that $\sigma\left(\mathbb{A}_{l}^{ \pm}\right)$is independent of $\lambda$. Moreover, since $\mathbb{F}^{ \pm}$is compact by Lemma 5.2, we find that the operator $\mathbb{A}_{l}^{ \pm}$is compact as well.

Now it follows easily from (5.12)-(5.13) that for each $\varepsilon \in(0,1)$ there exist subspaces $\mathscr{H}_{ \pm}$of $C_{0}^{\infty}(A, \infty)$ such that the codimensions codim $\mathscr{H}_{ \pm}$are finite and independent of $\lambda$, and

$$
\begin{array}{ll}
\left\|\widetilde{S}_{j}^{-}(\lambda ; A) u\right\|^{2} \geq(1+\varepsilon)^{-2}\left\|Q_{j}^{-}(\lambda ; A) u\right\|^{2}, & u \in \mathscr{H}_{-}, \\
\left\|\widetilde{S}_{j}^{+}(\lambda ; A) u\right\|^{2} \leq(1-\varepsilon)^{-2}\left\|Q_{j}^{+}(\lambda ; A) u\right\|^{2}, & u \in \mathscr{H}_{+} .
\end{array}
$$


Combining (5.16)-(5.17) with standard variational arguments (see, for instance, [2], Lemma 1.13, and the proof of Lemma 1.16 in [2]), we get

$$
\begin{aligned}
& n_{*}\left(r ; \widetilde{S}_{j}^{-}(\lambda ; A)\right) \geq n_{*}\left(r(1+\varepsilon) ; Q_{j}^{-}(\lambda ; A)\right)-\operatorname{codim} \mathscr{H}_{-}, \\
& n_{*}\left(r ; \widetilde{S}_{j}^{+}(\lambda ; A)\right) \leq n_{*}\left(r(1-\varepsilon) ; Q_{j}^{+}(\lambda ; A)\right)+\operatorname{codim} \mathscr{H}_{+} .
\end{aligned}
$$

Putting together (5.18)-(5.19) and (5.10), we arrive at (5.9).

Proposition 5.4. For every $r>0, A>0, \delta \in(0,1 / 2)$, and $\varepsilon \in(0,1)$, we have

$$
n_{*}\left(r ; Q_{j}^{-}(\lambda ; A)\right) \geq n_{*}\left(r(1+\varepsilon) \sqrt{W_{+}-W_{-}} ; \Gamma_{\delta}^{-}(\sqrt{b|\ln \lambda|})\right)+O(1)
$$

and

$$
\begin{aligned}
& n_{*}\left(r ; Q_{j}^{+}(\lambda ; A)\right) \\
& \quad \leq n_{*}\left(r(1-\varepsilon) \sqrt{W_{+}-W(-\delta)} e^{-b \delta^{2} / 2} ; \Gamma_{\delta}^{+}(\sqrt{b|\ln \lambda|})\right)+O(1),
\end{aligned}
$$

as $\lambda \downarrow 0$.

Proof. Let $\lambda>0, A \in[-\infty, \infty)$. Define the operators

$$
M_{j, 1}^{ \pm}(\lambda ; A): L^{2}\left(\Omega_{ \pm}\right) \rightarrow L^{2}\left(\Omega_{ \pm}\right)
$$

as the operators with integral kernels

$$
\begin{array}{r}
\frac{p_{j}}{2 \pi} e^{-b\left(x^{2}+x^{\prime 2}\right) / 2} \int_{A}^{\infty}\left(\mathcal{E}_{j}^{+}-E_{j}\left(k ; W_{0}^{ \pm}\right)+\lambda\right)^{-1} \\
k^{2(j-1)} e^{-b^{-1} k^{2}} e^{k\left(x+x^{\prime}+i\left(y-y^{\prime}\right)\right)} d k
\end{array}
$$

with $(x, y),\left(x^{\prime}, y^{\prime}\right) \in \Omega_{ \pm}$. Evidently, $Q_{j}^{ \pm}(\lambda ; A) Q_{j}^{ \pm}(\lambda ; A)^{*}=M_{j, 1}^{ \pm}(\lambda ; A)$. Therefore,

$$
n_{+}\left(r ; Q_{j}^{ \pm}(\lambda ; A)^{*} Q_{j}^{ \pm}(\lambda ; A)\right)=n_{+}\left(r ; M_{j, 1}^{ \pm}(\lambda ; A)\right), \quad r>0 .
$$

In the rest of the proof of the proposition we just show by successive simplifications that we can replace the operators $M_{j, 1}^{ \pm}(\lambda ; A)$ by their "asymptotic values" as $\lambda \downarrow 0$, namely the operators

$$
\text { const. } \Gamma_{\delta}^{ \pm}(\sqrt{b \mid \ln \lambda} \mid) \Gamma_{\delta}^{ \pm}(\sqrt{b \mid \ln \lambda} \mid)^{*} .
$$

The main ideas of these steps are inspired by the elementary asymptotic analysis as $\lambda \downarrow 0$ of the integral in (5.22); here we apply essentially relation (4.6) dealing with the asymptotics of $E_{j}(k)$ as $k \rightarrow \infty$. The technical details of the proof become somewhat tedious since we need to ensure an adequate control on the differences of the eigenvalue counting functions for the successive approximations. 
First, we concentrate at the proof of (5.20). Fix $\varepsilon>0$. Then by (4.6) there exists $A_{0}^{-}=A_{0}^{-}(\varepsilon)$ such that $k \geq A_{0}^{-}$implies

$$
\mathcal{E}_{j}^{+}-E_{j}\left(k ; W_{0}^{-}\right) \leq(1+\varepsilon) \frac{W_{+}-W_{-}}{2} p_{j} k^{2 j-3} e^{-b^{-1} k^{2}} .
$$

For $p>0$ and $A>0$ define $M_{j, 2}^{-}(\lambda, A, p): L^{2}\left(\Omega_{-}\right) \rightarrow L^{2}\left(\Omega_{-}\right)$as the operator with integral kernel

$$
\frac{p_{j}}{2 \pi} e^{-b\left(x^{2}+x^{\prime 2}\right) / 2} \int_{A}^{\infty}\left(p+\lambda k^{3-2 j} e^{b^{-1} k^{2}}\right)^{-1} k e^{k\left(x+x^{\prime}+i\left(y-y^{\prime}\right)\right)} d k
$$

with $(x, y),\left(x^{\prime}, y^{\prime}\right) \in \Omega_{-}$. Then (5.24) implies that for $A_{1}=\max \left\{A, A_{0}^{-}\right\}$we have

$$
n_{+}\left(r ; M_{j, 1}^{-}(\lambda ; A)\right) \geq n_{+}\left(r ; M_{j, 2}^{-}\left(\lambda, A_{1}, p_{j}(1+\varepsilon)\left(W_{+}-W_{-}\right) / 2\right)\right) .
$$

Fix $\delta \in(0,1 / 2)$. Set $\Lambda=|\ln \lambda|^{1 / 2}$, and assume that $\lambda>0$ is small that $A_{1}<\delta \sqrt{b} \Lambda$. Then, by the mini-max principle,

$$
n_{+}\left(r ; M_{j, 2}^{-}\left(\lambda, A_{1}, p\right)\right) \geq n_{+}\left(r ; M_{j, 2}^{-}(\lambda, \delta \sqrt{b} \Lambda, p)\right), \quad p>0, r>0 .
$$

In the integral defining the kernel of the operator $M_{j, 2}^{-}(\lambda, \delta \sqrt{b} \Lambda, p)$ (see (5.25)), change the variable $k=\sqrt{b} \Lambda(1+u)^{1 / 2}$ with $u \in\left(-1+\delta^{2}, \infty\right)$. Then we see that the integral kernel of $M_{j, 2}^{-}(\lambda, \delta \sqrt{b} \Lambda, p)$ is equal to

$$
\begin{array}{r}
\frac{p_{j} b \Lambda^{2}}{4 \pi} e^{-b\left(x^{2}+x^{\prime 2}\right) / 2} \int_{-1+\delta^{2}}^{\infty}\left(p+\left(\sqrt{b} \Lambda(1+u)^{1 / 2}\right)^{3-2 j} e^{\Lambda^{2} u}\right)^{-1} \\
e^{\left(x+x^{\prime}+i\left(y-y^{\prime}\right)\right) \sqrt{b} \Lambda(1+u)^{1 / 2}} d u .
\end{array}
$$

Define $M_{j, 3}^{-}(\lambda, \delta, p): L^{2}\left(\Omega_{-}\right) \rightarrow L^{2}\left(\Omega_{-}\right)$as the operator with integral kernel

$$
\begin{array}{r}
\frac{p_{j} b \Lambda^{2}}{4 \pi} e^{-b\left(x^{2}+x^{\prime 2}\right) / 2} \int_{-1+\delta^{2}}^{-1+(1-\delta)^{2}}\left(p+\left(\sqrt{b} \Lambda(1+u)^{1 / 2}\right)^{3-2 j} e^{\Lambda^{2} u}\right)^{-1} \\
e^{\left(x+x^{\prime}+i\left(y-y^{\prime}\right)\right) \sqrt{b} \Lambda(1+u)^{1 / 2}} d u
\end{array}
$$

with $(x, y),\left(x^{\prime}, y^{\prime}\right) \in \Omega_{-}$. Evidently, the mini-max principle implies

$$
n_{+}\left(r ; M_{j, 2}^{-}(\lambda, \delta \sqrt{b} \Lambda, p)\right) \geq n_{+}\left(r ; M_{j, 3}^{-}(\lambda, \delta, p)\right),
$$

for $p>0, r>0$, and $\delta \in(0,1 / 2)$. Further, define

$$
M_{j, 4}^{-}(\lambda, \delta, p): L^{2}\left(\Omega_{-}\right) \rightarrow L^{2}\left(\Omega_{-}\right)
$$

as the operator with integral kernel

$$
\frac{p_{j} b \Lambda^{2}}{4 \pi p} e^{-b\left(x^{2}+x^{\prime 2}\right) / 2} \int_{-1+\delta^{2}}^{-1+(1-\delta)^{2}} e^{\left(x+x^{\prime}+i\left(y-y^{\prime}\right)\right) \sqrt{b} \Lambda(1+u)^{1 / 2}} d u
$$


with $(x, y),\left(x^{\prime}, y^{\prime}\right) \in \Omega_{-}$. By the dominated convergence theorem,

$$
\lim _{\lambda \downarrow 0}\left\|M_{j, 3}^{-}(\lambda, \delta, p)-M_{j, 4}^{-}(\lambda, \delta, p)\right\|_{2}^{2}=0
$$

where $\|\cdot\|_{2}$ denotes the Hilbert-Schmidt norm. Fix $\varepsilon>0$. Applying the Weyl inequalities and the elementary Chebyshev-type estimate

$n_{*}\left(s ; M_{j, 3}^{-}(\lambda, \delta, p)-M_{j, 4}^{-}(\lambda, \delta, p)\right) \leq s^{-2}\left\|M_{j, 3}^{-}(\lambda, \delta, p)-M_{j, 4}^{-}(\lambda, \delta, p)\right\|_{2}^{2}, \quad s>0$, we get

$$
n_{+}\left(r ; M_{j, 3}^{-}(\lambda, \delta, p)\right) \geq n_{+}\left(r(1+\varepsilon) ; M_{j, 4}^{-}(\lambda, \delta, p)\right)+O(1), \quad \lambda \downarrow 0 .
$$

In the integral defining the kernel of the operator $M_{j, 4}^{-}(\lambda, \delta, 1)$, see (5.29), change the variable $(1+u)^{1 / 2}=k$ with $k \in(\delta, 1-\delta)$. Then we see that the integral kernel of $M_{j, 4}^{-}(\lambda, \delta, 1)$ equals

$$
\frac{p_{j} b \Lambda^{2}}{2 \pi p} e^{-b\left(x^{2}+x^{\prime 2}\right) / 2} \int_{\delta}^{1-\delta} e^{\left(x+x^{\prime}+i\left(y-y^{\prime}\right)\right) \sqrt{b} \Lambda k} k d k, \quad(x, y),\left(x^{\prime}, y^{\prime}\right) \in \Omega_{-} .
$$

Therefore

$$
M_{j, 4}^{-}(\lambda, \delta, p)=\frac{p_{j}}{2 p} \Gamma_{\delta}^{-}(\sqrt{b|\ln \lambda|}) \Gamma_{\delta}^{-}(\sqrt{b|\ln \lambda|})^{*}
$$

Combining now (5.23), (5.26), (5.27), (5.28), (5.30), and (5.31), we obtain (5.20).

Let us now prove (5.21). The proof is quite similar to that of (5.20), so that we omit certain details. Set $v_{1}=0$ and $v_{j}=1$ if $j \in \mathbb{N}, j \geq 2$. Pick $\varepsilon \in(0,1)$. Then there exists $A_{0}^{+}=A_{0}^{+}(\varepsilon)$ such that $k \geq A_{0}^{+}$implies

$$
\varepsilon_{j}^{+}-E_{j}\left(k ; W_{0}^{+}\right) \geq(1-\varepsilon) \frac{W_{+}-W(-\delta)}{2} p_{j}\left(k+v_{j}\right)^{2 j-3} e^{-\left(b^{-1 / 2} k+b^{1 / 2} \delta\right)^{2}} \text {. }
$$

For $p>0$ and $A>0$ define $M_{j, 2}^{+}(\lambda, A, p): L^{2}\left(\Omega_{+}\right) \rightarrow L^{2}\left(\Omega_{+}\right)$as the operator with integral kernel

$$
\begin{array}{r}
\frac{p_{j}}{2 \pi} e^{-b\left(x^{2}+x^{\prime 2}\right) / 2} \int_{A}^{\infty}\left(p+\lambda\left(k+v_{j}\right)^{3-2 j} e^{b^{-1} k^{2}+2 \delta k}\right)^{-1} k \\
e^{k\left(x+x^{\prime}+i\left(y-y^{\prime}\right)+2 \delta\right)} d k
\end{array}
$$

with $(x, y),\left(x^{\prime}, y^{\prime}\right) \in \Omega_{+}$. Therefore, similarly to (5.26), we have

$$
\begin{aligned}
& n_{+}\left(r ; M_{j, 1}^{+}(\lambda ; A)\right) \\
& \quad \leq n_{+}\left(r ; M_{j, 2}^{+}\left(\lambda, A_{1},(1-\varepsilon) p_{j} e^{-b \delta^{2}}\left(W_{+}-W(-\delta)\right) / 2\right)\right)
\end{aligned}
$$

for $A_{1}=\max \left\{A, A_{0}^{+}\right\}$. Moreover, it is easy to check that

$$
n_{+}\left(r ; M_{j, 2}^{+}(\lambda, A, p)\right)=n_{+}\left(r ; M_{j, 2}^{+}(\lambda, 0, p)\right)+O(1), \quad \lambda \downarrow 0,
$$


for any $A \geq 0, p>0$. In the integral defining the kernel of the operator $M_{j, 2}^{+}(\lambda, 0, p)$ (see (5.33)), change the variable $k=\sqrt{b} \Lambda(1+u)^{1 / 2}$ with $u \in(-1, \infty)$. Then we see that the integral kernel of $M_{j, 2}^{+}(\lambda, 0, p)$ is equal to

$$
\begin{array}{r}
\frac{p_{j} b \Lambda^{2}}{4 \pi} e^{-b\left(x^{2}+x^{\prime 2}\right) / 2} \int_{-1}^{\infty}\left(p+\left(\sqrt{b} \Lambda(1+u)^{1 / 2}+v_{j}\right)^{3-2 j} e^{\Lambda^{2} u+2 \delta \sqrt{b} \Lambda(1+u)^{1 / 2}}\right)^{-1} \\
e^{\left(x+x^{\prime}+i\left(y-y^{\prime}\right)+2 \delta\right) \sqrt{b} \Lambda(1+u)^{1 / 2}} d u .
\end{array}
$$

Define now $M_{j, 3}^{+}(\lambda, \delta, p): L^{2}\left(\Omega_{+}\right) \rightarrow L^{2}\left(\Omega_{+}\right)$, as the operator with integral kernel

$$
\begin{gathered}
\frac{p_{j} b \Lambda^{2}}{4 \pi} e^{-b\left(x^{2}+x^{\prime 2}\right) / 2} \int_{-1}^{-1+(1+\delta)^{2}}\left(p+\left(\sqrt{b} \Lambda(1+u)^{1 / 2}+v_{j}\right)^{3-2 j}\right. \\
e^{\left.\Lambda^{2} u+2 \delta \sqrt{b} \Lambda(1+u)^{1 / 2}\right)^{-1}} \\
e^{\left(x+x^{\prime}+i\left(y-y^{\prime}\right)+2 \delta\right) \sqrt{b} \Lambda(1+u)^{1 / 2}} d u
\end{gathered}
$$

with $(x, y),\left(x^{\prime}, y^{\prime}\right) \in \Omega_{+}$. By the dominated convergence theorem,

$$
\lim _{\lambda \downarrow 0}\left\|M_{j, 2}^{+}(\lambda, 0, p)-M_{j, 3}^{+}(\lambda, \delta, p)\right\|_{2}^{2}=0 .
$$

Therefore, similarly to (5.30), we obtain

$$
n_{+}\left(r ; M_{j, 2}^{+}(\lambda, 0, p)\right) \leq n_{+}\left(r(1-\varepsilon) ; M_{j, 3}^{+}(\lambda, \delta, p)\right)+O(1), \quad \lambda \downarrow 0,
$$

for any $r>0, \varepsilon \in(0,1), \delta>0, p>0$. Next, define

$$
M_{j, 4}^{+}(\lambda, \delta, p): L^{2}\left(\Omega_{+}\right) \rightarrow L^{2}\left(\Omega_{+}\right), \quad \delta>0,
$$

as the operator with integral kernel

$$
\frac{b p_{j} \Lambda^{2}}{4 \pi p} e^{-b\left(x^{2}+x^{\prime 2}\right) / 2} \int_{-1}^{-1+(1+\delta)^{2}} e^{\left(x+x^{\prime}+i\left(y-y^{\prime}\right)+2 \delta\right) \sqrt{b} \Lambda(1+u)^{1 / 2}} d u,
$$

for $(x, y),\left(x^{\prime}, y^{\prime}\right) \in \Omega_{+}$. Evidently, the mini-max principle implies

$$
n_{+}\left(r ; M_{j, 3}^{+}(\lambda, \delta, p)\right) \leq n_{+}\left(r ; M_{j, 4}^{+}(\lambda, \delta, p)\right), \quad r>0 .
$$

Finally, by analogy with (5.31), we get

$$
M_{j, 4}^{+}(\lambda, \delta, p)=\frac{p_{j}}{2 p} \Gamma_{\delta}^{+}(\sqrt{b|\ln \lambda|}) \Gamma_{\delta}^{+}(\sqrt{b|\ln \lambda|})^{*} .
$$

Putting together (5.23) and (5.34)-(5.38), we arrive at (5.20).

Now, the combination of (5.9) and (5.20)-(5.21) yields (5.3)-(5.4). 


\section{Asymptotic bounds of $\mathcal{N}_{j}^{+}(\lambda)$ as $\lambda \downarrow 0$}

In what follows we identify when appropriate $\mathbb{R}^{2}$ with $\mathbb{C}$ writing $z=x+i y \in \mathbb{C}$ for $(x, y) \in \mathbb{R}^{2}$. Moreover, we denote by $d \mu(z)=d x d y$ the Lebesgue measure on $\mathbb{R}^{2}$. Further, we assume as before that $x^{+}=0$, that $V$ satisfies (5.1) with some constants $c_{0}^{ \pm}>0$ and some bounded domains $\Omega_{ \pm} \subset \mathbb{R}^{2}$ with Lipschitz boundaries, and that

$$
\Omega_{-} \cap\{z \in \mathbb{C} \mid \operatorname{Re} z>0\} \neq \emptyset .
$$

We will show that under these assumptions the functions $\mathcal{N}_{j}^{+}(\lambda)$ satisfy the asymptotic estimates (1.6) and (1.7) with some explicit constants $\boldsymbol{C}_{ \pm}>0$. In order to define these constants we need the following notations. Let $\Omega \subset \mathbb{R}^{2}$ be a bounded domain. Put

$$
\begin{array}{r}
K_{-}(\Omega)=\left\{(p, q) \in \mathbb{R}^{2} \mid p<q \text { and there exists } x \in \mathbb{R}\right. \text { such that } \\
(x, p+t(q-p)) \in \Omega \text { for all } t \in[0,1]\},
\end{array}
$$

and

$$
\mathbf{c}_{-}(\Omega)=\sup _{(p, q) \in K_{-}(\Omega)}(q-p) .
$$

In other words, $\mathbf{c}_{-}(\Omega)$ is just the maximal length of the vertical segments contained in $\bar{\Omega}$. Next, for $s \in[0, \infty)$ put

$$
\varkappa(s)=|\{t>0 \mid t \ln t<s\}|
$$

where $|\cdot|$ denotes the Lebesgue measure of a Borel set in $\mathbb{R}$. Let $B_{R}(\zeta) \subset \mathbb{R}^{2}$ be the open disk of radius $R>0$ centered at $\zeta \in \mathbb{C}$. Set

$$
K_{+}(\Omega)=\left\{(\xi, R) \in \mathbb{R} \times(0, \infty) \mid \text { there exists } \eta \in \mathbb{R} \text { such that } \Omega \subset B_{R}(\xi+i \eta)\right\},
$$

and

$$
\mathbf{c}_{+}(\Omega)=\inf _{(\xi, R) \in K_{+}(\Omega)} R \varkappa\left(\frac{\xi_{+}}{e R}\right)
$$

where $\xi_{+}=\max \{\xi, 0\}$. Evidently,

$$
\mathbf{c}_{+}(\Omega) \geq \frac{1}{2} \operatorname{diam}(\Omega) \geq \frac{1}{2} \mathbf{c}_{-}(\Omega) .
$$

Finally, put

$$
\widetilde{\Omega}_{ \pm}=\left\{z \in \Omega_{ \pm} \mid \operatorname{Re} z>0\right\} .
$$

Note that (6.1) implies $\widetilde{\Omega}_{ \pm} \neq \emptyset$. Occasionally, we will also use the notation

$$
\widetilde{\Omega}_{+}(\delta)=\left\{z \in \Omega_{+} \mid \operatorname{Re} z>-2 \delta\right\}
$$

for $\delta \geq 0$ so that $\widetilde{\Omega}_{+}(0)=\tilde{\Omega}_{+}$. 
Theorem 6.1. Suppose that $W$ is a bounded non-decreasing function with $W_{-}<W_{+}$, and $x^{+}=0$. Assume that $V$ satisfies (5.1), and (6.1) holds true. Then asymptotic relation (1.6) is satisfied with $\boldsymbol{C}_{-}=(2 \pi)^{-1} \sqrt{b} \mathbf{c}_{-}\left(\widetilde{\Omega}_{-}\right)$while asymptotic relation (1.7) holds true with $\mathcal{C}_{+}=e \sqrt{b} \mathbf{c}_{+}\left(\widetilde{\Omega}_{+}\right)$. In particular,

$$
\lim _{\lambda \downarrow 0} \frac{\ln \mathcal{N}_{j}^{+}(\lambda)}{\ln |\ln \lambda|}=\frac{1}{2}, \quad j \in \mathbb{N} .
$$

Remark. Under the hypotheses of Theorem 6.1 we have $\ell_{-}<\ell_{+}$due to (6.2), $\widetilde{\Omega}_{-} \subset \widetilde{\Omega}_{+}$, and $1 / \pi<e$.

The proof of (1.6) is contained in Subsection 6.1, and the proof of (1.7) can be found in Subsection 6.2.

6.1. Lower bound of $\mathcal{N}_{j}^{+}(\lambda)$. In this subsection we prove (1.6). Taking into account Theorem 3.2, (3.17), and Theorem 5.1, we find that it suffices to show that, for any $r>0$ independent of $\lambda>0$, we have

$$
\lim _{\delta \downarrow 0} \liminf _{\lambda \downarrow 0}|\ln \lambda|^{-1 / 2} n_{+}\left(r ; \Gamma_{\delta}^{-}(\sqrt{b|\ln \lambda|})^{*} \Gamma_{\delta}^{-}(\sqrt{b|\ln \lambda|})\right) \geq \mathcal{C}_{-} .
$$

Let $\Omega \subset \mathbb{R}^{2}$ be a bounded domain, and $d \subset(0, \infty)$ be a bounded open non-empty interval. For $m>0$ and $\delta \geq 0$ define the operator $\mathscr{E}_{m, \delta}(\Omega, \ell): L^{2}(\ell) \rightarrow L^{2}(\ell)$ as the operator with integral kernel

$$
\pi^{-1} m^{2} \sqrt{k k^{\prime}} \int_{\Omega} e^{m\left((z+\delta) k+(\bar{z}+\delta) k^{\prime}\right)} d \mu(z), \quad k, k^{\prime} \in \mathcal{d} .
$$

Set

$$
\varepsilon_{-}=\inf _{x \in \omega_{-}} e^{-b x^{2}}, \quad \varepsilon_{+}=\sup _{x \in \omega_{+}} e^{-b x^{2}},
$$

the sets $\omega_{ \pm}$being defined in (5.7). Then we have

$$
\Gamma_{\delta}^{-}(m)^{*} \Gamma_{\delta}^{-}(m) \geq \varepsilon_{-} \mathcal{E}_{m, 0}\left(\Omega_{-}, I_{-}(\delta)\right), \quad m>0 .
$$

Further, let $\mathcal{R} \subset \widetilde{\Omega}_{-} \subset \Omega_{-}$be an open non-empty rectangle whose sides are parallel to the coordinate axes. Since a translation $z \mapsto z+i \eta, \eta \in \mathbb{R}$, in the integral in (6.4) generates a unitary transformation of the operator $\mathscr{S}_{m, 0}\left(\Omega_{-}, I_{-}(\delta)\right)$ into an operator unitarily equivalent to it, we assume without any loss of generality that $\mathcal{R}=(\alpha, \beta) \times(-L, L)$ with $0<\alpha<\beta<\infty$ and $L \in(0, \infty)$. Evidently,

$$
\mathscr{E}_{m, 0}\left(\Omega_{-}, I_{-}(\delta)\right) \geq \mathscr{E}_{m, 0}\left(\mathcal{R}, I_{-}(\delta)\right), \quad m>0 .
$$

For $\eta \in \mathbb{R}$ and $\delta \in(0,1 / 2)$ define the operator $G_{\eta, \delta}^{-}(m): L^{2}\left(I_{-}(\delta)\right) \rightarrow L^{2}\left(I_{-}(\delta)\right)$ as the integral operator with kernel

$$
e^{\eta m\left(k+k^{\prime}\right)} \frac{\sin \left(m\left(k-k^{\prime}\right)\right)}{\pi\left(k-k^{\prime}\right)} \frac{2 \sqrt{k k^{\prime}}}{k+k^{\prime}}, \quad k, k^{\prime} \in I_{-}(\delta) .
$$


Then

$$
\mathscr{E}_{m, 0}\left(\mathcal{R}, I_{-}(\delta)\right)=G_{\beta, \delta}^{-}(m L)-G_{\alpha, \delta}^{-}(m L) .
$$

Define the operator $g_{\ell}(m): L^{2}(\mathcal{l}) \rightarrow L^{2}(\mathcal{l}), m>0$, as the operator with integral kernel

$$
\frac{\sin \left(m\left(k-k^{\prime}\right)\right)}{\pi\left(k-k^{\prime}\right)} \frac{2 \sqrt{k k^{\prime}}}{k+k^{\prime}}, \quad k, k^{\prime} \in \mathcal{d} .
$$

Note that we have $g_{d}(m)=\gamma_{d}(m)^{*} \gamma_{d}(m)$ where

$$
\gamma_{\mathcal{Q}}(m): L^{2}(\mathcal{\ell}) \rightarrow L^{2}((0, \infty) \times(-m, m))
$$

is the operator with integral kernel

$$
\pi^{-1 / 2} e^{-(x+i y) k} k^{1 / 2}, \quad k \in \mathcal{l}, x \in(0, \infty), y \in(-m, m) .
$$

Evidently, for any finite $m>0$, the operator $\gamma_{\mathcal{l}}(m)$ is Hilbert-Schmidt, and we have $\left\|\gamma_{\ell}(m)\right\|<1$. Therefore, $g_{\ell}(m)=g_{\ell}(m)^{*} \geq 0$ is a trace-class operator, and

$$
\left\|g_{\ell}(m)\right\|<1 .
$$

Simple variational arguments yield

$$
\begin{aligned}
n_{+}(r & \left.; G_{\beta, \delta}^{-}(m)-G_{\alpha, \delta}^{-}(m)\right) \\
& \geq n_{+}\left(r\left(1-e^{2(\alpha-\beta) \delta m}\right)^{-1} ; G_{\beta, \delta}^{-}(m)\right) \\
& \geq n_{+}\left(r e^{-2 \beta \delta m}\left(1-e^{2(\alpha-\beta) \delta m}\right)^{-1} ; g_{I_{-}(\delta)}(m)\right),
\end{aligned}
$$

for $r>0$ and $\delta \in(0,1 / 2)$. Combining (6.6)-(6.10), we find that under the hypotheses of Theorem 6.1 for each $\delta \in(0,1 / 2)$ we have

$$
\begin{aligned}
& n_{+}\left(r ; \Gamma_{\delta}^{-}(m)^{*} \Gamma_{\delta}^{-}(m)\right) \\
& \quad \geq n_{+}\left(r e^{-2 \beta \delta m}\left(\varepsilon_{-}\left(1-e^{2(\alpha-\beta) \delta m}\right)\right)^{-1} ; g_{I_{-}(\delta)}(m L)\right) .
\end{aligned}
$$

In order to complete the proof of (6.3), we need the following result.

Proposition 6.2. For all $l \in \mathbb{N}$ we have

$$
\lim _{m \rightarrow \infty} m^{-1} \operatorname{Tr} g_{\ell}(m)^{l}=\frac{|\ell|}{\pi} .
$$

Proof. Let $l=1$. Then, $\operatorname{Tr} g_{\ell}(m)=\frac{m|\ell|}{\pi}$. Let now $l \geq 2$. Set

$$
\varphi_{m}(k)=\frac{\sin m k}{\pi k}, \quad k \in d .
$$

Denote by $\chi_{\ell}$ the characteristic function of the interval $\ell$. Then we have 


$$
\begin{array}{r}
\operatorname{Tr} g_{\ell}(m)^{l}=\int_{\mathbb{R}} \ldots \int_{\mathbb{R}} \varphi_{m}\left(k_{1}-k_{2}\right) \varphi_{m}\left(k_{2}-k_{3}\right) \ldots \varphi_{m}\left(k_{l-1}-k_{l}\right) \varphi_{m}\left(k_{l}-k_{1}\right) \\
\mathbb{G}_{l}\left(k_{1}, \ldots, k_{l}\right) \chi_{\ell}\left(k_{1}\right) \ldots \chi_{\ell}\left(k_{l}\right) d k_{1} \ldots d k_{l},
\end{array}
$$

where

$$
\mathbb{G}_{l}\left(k_{1}, \ldots, k_{l}\right)=\frac{2^{l} k_{1} \ldots k_{l}}{\left(k_{1}+k_{2}\right)\left(k_{2}+k_{3}\right) \ldots\left(k_{l-1}+k_{l}\right)\left(k_{l}+k_{1}\right)} .
$$

Changing the variables $k_{1}=t_{1}, k_{j}=t_{1}+m^{-1} t_{j}, j=2, \ldots, l$, we get

$$
\begin{aligned}
\operatorname{Tr} g_{\ell}(m)^{l} & =m \int_{\mathbb{R}} \ldots \int_{\mathbb{R}} \varphi_{1}\left(-t_{2}\right) \varphi_{1}\left(t_{2}-t_{3}\right) \ldots \varphi_{1}\left(t_{l-1}-t_{l}\right) \varphi_{1}\left(t_{l}\right) \\
& \widetilde{\mathbb{G}}_{l, m}\left(t_{1}, \ldots, t_{l}\right) \chi_{\ell}\left(t_{1}\right) \chi_{\ell}\left(t_{1}+m^{-1} t_{2}\right) \ldots \chi_{\ell}\left(t_{1}+m^{-1} t_{l}\right) d t_{1} \ldots d t_{l},
\end{aligned}
$$

where

$$
\begin{gathered}
\widetilde{\mathbb{G}}_{l, m}\left(t_{1}, \ldots, t_{l}\right)= \\
\frac{2^{l} t_{1}\left(t_{1}+m^{-1} t_{2}\right) \ldots\left(t_{1}+m^{-1} t_{l}\right)}{\left(2 t_{1}+m^{-1} t_{2}\right)\left(2 t_{1}+m^{-1}\left(t_{2}+t_{3}\right)\right) \ldots\left(2 t_{1}+m^{-1}\left(t_{l-1}+t_{l}\right)\right)\left(2 t_{1}+m^{-1} t_{l}\right)} .
\end{gathered}
$$

Applying the dominated convergence theorem, we get

$$
\begin{aligned}
& \lim _{m \rightarrow \infty} m^{-1} \operatorname{Tr} g_{\ell}(m)^{l} \\
& \quad=|\ell| \int_{\mathbb{R}} \ldots \int_{\mathbb{R}} \varphi_{1}\left(-t_{2}\right) \varphi_{1}\left(t_{2}-t_{3}\right) \ldots \varphi_{1}\left(t_{l-1}-t_{l}\right) \varphi_{1}\left(t_{l}\right) d t_{2} \ldots d t_{l} .
\end{aligned}
$$

Further, we have

$$
\varphi_{1}(t)=\frac{1}{2 \pi} \int_{\mathbb{R}} e^{i t \xi} \chi_{(-1,1)}(\xi) d \xi, \quad t \in \mathbb{R} .
$$

Therefore,

$$
\begin{aligned}
\int_{\mathbb{R}} \ldots & \int_{\mathbb{R}} \varphi_{1}\left(-t_{2}\right) \varphi_{1}\left(t_{2}-t_{3}\right) \ldots \varphi_{1}\left(t_{l-1}-t_{l}\right) \varphi_{1}\left(t_{l}\right) d t_{2} \ldots d t_{l} \\
& =\frac{1}{2 \pi} \int_{\mathbb{R}} \chi_{(-1,1)}(\xi)^{l} d \xi=\frac{1}{\pi} .
\end{aligned}
$$

Putting together (6.13) and (6.14), we obtain (6.12).

Proposition 6.2 and estimate (6.9) combined with the Kac-Murdock-Szegó theorem (see the original work [12], [8], Section 11.8, or [22], Lemmas 3.1, and 3.2), imply the following result. 
Corollary 6.3. We have

$$
\lim _{m \rightarrow \infty} m^{-1} n_{+}\left(s ; g_{\ell}(m)\right)= \begin{cases}\frac{|\mathcal{}|}{\pi} & \text { if } s \in(0,1), \\ 0 & \text { if } s>1 .\end{cases}
$$

Now we are in position to prove (6.3). Fix arbitrary $s \in(0,1)$. Assume that $m$ is so large that $r e^{-2 \beta \delta m}\left(\varepsilon_{-}\left(1-e^{2(\alpha-\beta) \delta m}\right)\right)^{-1}<s$. Then (6.11) implies

$$
n_{+}\left(r ; \Gamma_{\delta}^{-}(m)^{*} \Gamma_{\delta}^{-}(m)\right) \geq n_{+}\left(s ; g_{I_{-}(\delta)}(m L)\right) .
$$

Putting together (6.15) and (6.16), we find that the asymptotic estimate

$$
\liminf _{\lambda \downarrow 0}|\ln \lambda|^{-1 / 2} n_{+}\left(r ; \Gamma_{\delta}^{-}(\sqrt{b|\ln \lambda|})^{*} \Gamma_{\delta}^{-}(\sqrt{b|\ln \lambda|})\right) \geq \frac{\sqrt{b} L}{\pi}(1-2 \delta)
$$

holds for every $\delta \in(0,1 / 2)$. Letting $\delta \downarrow 0$, and optimizing with respect to $L$ we obtain (6.3).

6.2. Upper bound of $\mathcal{N}_{j}^{+}(\lambda)$. In this subsection we prove (1.7). By analogy with (6.3), it suffices to show that for any $r>0$ independent of $\lambda>0$, we have

$$
\lim _{\delta \downarrow 0} \limsup _{\lambda \downarrow 0}|\ln \lambda|^{-1 / 2} n_{+}\left(r ; \Gamma_{\delta}^{+}(\sqrt{b|\ln \lambda|})^{*} \Gamma_{\delta}^{+}(\sqrt{b|\ln \lambda|})\right) \leq \iota_{+} .
$$

Evidently,

$$
\Gamma_{\delta}^{+}(m)^{*} \Gamma_{\delta}^{+}(m) \leq \varepsilon_{+} \mathcal{E}_{m, \delta}\left(\Omega_{+} ; I_{+}(\delta)\right), \quad m>0,
$$

the integral kernel of the operator $\mathscr{E}_{m, \delta}\left(\Omega_{+} ; \ell\right)$ being defined in (6.4), and the number $\varepsilon_{+}$being defined in (6.5). Since we have $\mathcal{E}_{m, \delta}\left(\Omega_{+} \backslash \widetilde{\Omega}_{+}(\delta) ; I_{+}(\delta)\right) \geq 0$ and

$$
\begin{aligned}
\lim _{m \rightarrow \infty} \operatorname{Tr} \mathscr{E}_{m, \delta}\left(\Omega_{+} \backslash \tilde{\Omega}_{+}(\delta) ; I_{+}(\delta)\right) \\
\quad=\pi^{-1} \lim _{m \rightarrow \infty} m^{2} \int_{0}^{1+\delta} \int_{\Omega_{+} \backslash \tilde{\Omega}_{+}(\delta)} e^{2 m(\operatorname{Re} z+\delta) k} d \mu(z) k d k=0,
\end{aligned}
$$

we easily find that the Weyl inequalities entail

$$
\begin{aligned}
& n_{+}\left(r ; \mathscr{\mathcal { E }}_{m, \delta}\left(\Omega_{+} ; I_{+}(\delta)\right)\right) \\
& \quad \leq n_{+}\left(r(1-\varepsilon) ; \mathscr{I}_{m, \delta}\left(\widetilde{\Omega}_{+}(\delta) ; I_{+}(\delta)\right)\right)+O(1), \quad m \rightarrow \infty,
\end{aligned}
$$

for each $r>0$ and $\varepsilon \in(0,1)$. Further, pick an open disk $B_{R}(\zeta) \subset \mathbb{R}^{2}$ such that $\widetilde{\Omega}_{+}(\delta) \subset B_{R}(\zeta)$. Evidently,

$$
n_{+}\left(r ; \boldsymbol{E}_{m, \delta}\left(\widetilde{\Omega}_{+}(\delta) ; I_{+}(\delta)\right)\right) \leq n_{+}\left(r ; \boldsymbol{E}_{m, \delta}\left(B_{R}(\zeta) ; I_{+}(\delta)\right)\right), \quad r>0 .
$$


Next, put $I_{*}=I_{*}(\delta)=\left(0,(1+\delta)^{-1}\right)$, and define

$$
G_{\delta}^{+}(m): L^{2}\left(I_{*}\right) \rightarrow L^{2}\left(I_{*}\right)
$$

as the operator with integral kernel

$$
\pi^{-1} m^{2} e^{2 m(\xi+\delta)+} \int_{B_{R}(0)} e^{m\left(z k+\bar{z} k^{\prime}\right)} d \mu(z), \quad k, k^{\prime} \in I_{*}(\delta) .
$$

Changing the variable

$$
z \mapsto z+\zeta
$$

in the integral defining the kernel of $\mathscr{E}_{m, \delta}\left(B_{R}(\zeta) ; I_{+}(\delta)\right)$, see (6.4), and after that changing the variable

$$
k \mapsto(1+\delta)^{2} k
$$

in $I_{*}(\delta)$, we find that the mini-max principle implies

$$
n_{+}\left(r ; \mathscr{E}_{m, \delta}\left(B_{R}(\zeta) ; I_{+}(\delta)\right)\right) \leq n_{+}\left(r ; G_{\delta}^{+}\left((1+\delta)^{2} m\right)\right), \quad r>0,
$$

with $\xi=\operatorname{Re} \zeta$. Further, expanding the exponential functions into power series, and passing to polar coordinates, we get

$$
\int_{B_{R}(0)} e^{m\left(z k+\bar{z} k^{\prime}\right)} d \mu(z)=\pi R^{2} \sum_{q=0}^{\infty} \frac{\left(m^{2} R^{2} k k^{\prime}\right)^{q}}{(q !)^{2}(q+1)} .
$$

Therefore, the quadratic form of the operator $G_{\delta}^{+}(m)$ can be written as

$$
\left\langle G_{\delta}^{+}(m) u, u\right\rangle_{L^{2}\left(I_{*}\right)}=e^{2 m(\xi+\delta)_{+}} \sum_{q=0}^{\infty} \frac{(m R)^{2 q+2}}{(q !)^{2}(q+1)}\left|\tilde{u}_{q}\right|^{2}
$$

where

$$
\tilde{u}_{q}=\int_{I_{*}(\delta)} k^{q} u(k) d k, \quad u \in L^{2}\left(I_{*}(\delta)\right), \quad q \in \mathbb{Z}_{+} .
$$

Let $\left\{p_{q}(k)\right\}_{q \in \mathbb{Z}_{+}}$be the system of polynomials orthonormal in $L^{2}\left(I_{*}(\delta)\right)$, obtained by the Gram-Schmidt procedure from $\left\{k^{q}\right\}_{q \in \mathbb{Z}_{+}}, k \in I_{*}(\delta)$. Then,

$$
k^{q}=\sum_{l=0}^{q} \theta_{q, l} p_{l}(k), \quad k \in I_{*}(\delta), q \in \mathbb{Z}_{+},
$$

with appropriate $\theta_{q, l}$; in what follows we set $\theta_{q, l}=0$ for $l>q$. Put

$$
u_{q}=\int_{I_{*}(\delta)} p_{q}(k) u(k) d k, \quad u \in L^{2}\left(I_{*}(\delta)\right), \quad q \in \mathbb{Z}_{+} .
$$


Then we have

$$
\tilde{u}_{q}=\sum_{l=0}^{\infty} \theta_{q, l} u_{l}, \quad q \in \mathbb{Z}_{+}
$$

and

$$
\|u\|_{L^{2}\left(I_{*}(\delta)\right)}^{2}=\sum_{q=0}^{\infty}\left|u_{q}\right|^{2} .
$$

Further, it is easy to check that

$$
\sum_{q=0}^{\infty} \sum_{l=0}^{\infty} \theta_{q, l}^{2}=\sum_{l=0}^{\infty} \int_{I_{*}(\delta)} k^{2 l} d k=\sum_{l=0}^{\infty} \frac{(1+\delta)^{-2 l-1}}{2 l+1}<\infty .
$$

Therefore, the operator $\Theta: l^{2}\left(\mathbb{Z}_{+}\right) \rightarrow l^{2}\left(\mathbb{Z}_{+}\right)$defined by

$$
(\Theta \mathbf{u})_{q}=\sum_{l=0}^{\infty} \theta_{q, l} u_{l}, \quad q \in \mathbb{Z}_{+}, \quad \mathbf{u}=\left\{u_{l}\right\}_{l \in \mathbb{Z}_{+}},
$$

is a Hilbert-Schmidt, and hence bounded operator. Let $\rho(m): l^{2}\left(\mathbb{Z}_{+}\right) \rightarrow l^{2}\left(\mathbb{Z}_{+}\right)$ be the diagonal operator with diagonal entries

$$
e^{2 m(\xi+\delta)}+\frac{(m R)^{2 q+2}}{(q !)^{2}(q+1)}, \quad q \in \mathbb{Z}_{+} .
$$

Now (6.22)-(6.25) imply

$$
n_{+}\left(s ; G_{\delta}^{+}(m)\right)=n_{+}\left(s ; \Theta^{*} \rho(m) \Theta\right), \quad s>0 .
$$

Evidently,

$$
n_{+}\left(s ; \Theta^{*} \rho(m) \Theta\right) \leq n_{+}\left(s ;\|\Theta\|^{2} \rho(m)\right), \quad s>0 .
$$

On the other hand, for any $s>0$ we have

$$
n_{+}(s ; \rho(m))=\#\left\{q \in \mathbb{Z}_{+} \mid \frac{e^{m(\xi+\delta)_{+}}(m R)^{q+1}}{q ! \sqrt{q+1}}>\sqrt{s}\right\}, \quad s>0 .
$$

Applying the Stirling formula

$$
q !=(2 \pi)^{1 / 2}(q+1)^{q+1}(q+1)^{-1 / 2} e^{-q-1}(1+o(1)), \quad q \rightarrow \infty,
$$

we find that for each $\varepsilon \in(0,1)$ there exists $q_{0} \in \mathbb{Z}_{+}$such that

$$
\begin{gathered}
\left\{q \in \mathbb{Z}_{+} \mid \frac{e^{m(\xi+\delta)_{+}}(m R)^{q+1}}{q ! \sqrt{q+1}}>\sqrt{s}\right\} \leq \\
\#\left\{q \in \mathbb{Z}_{+} \mid \frac{(\xi+\delta)_{+}}{e R}>\frac{q+1}{e R m} \ln \left(\frac{q+1}{e R m}\right)+\frac{\ln (\sqrt{2 \pi s}(1-\varepsilon))}{e R m}\right\}+q_{0} .
\end{gathered}
$$


Passing from Darboux sums to Riemann integrals, we find that for each constant $c \in \mathbb{R}$ we have

$$
\begin{aligned}
\lim _{m \rightarrow \infty} m^{-1} \#\left\{q \in \mathbb{Z}_{+} \mid \frac{(\xi+\delta)_{+}}{e R}>\frac{q+1}{e R m} \ln \left(\frac{q+1}{e R m}\right)+\frac{c}{m}\right\} \\
=e R \varkappa\left(\frac{(\xi+\delta)_{+}}{e R}\right) .
\end{aligned}
$$

Putting together (6.18)-(6.21) and (6.26)-(6.30), we get

$$
\begin{aligned}
& \limsup _{\lambda \downarrow 0}|\ln \lambda|^{-1 / 2} n_{+}\left(r ; \Gamma_{\delta}^{+}(\sqrt{b|\ln \lambda|})^{*} \Gamma_{\delta}^{+}(\sqrt{b|\ln \lambda|})\right) \\
& \quad \leq(1+\delta)^{2} \sqrt{b} e R \varkappa\left(\frac{(\xi+\delta)_{+}}{e R}\right)
\end{aligned}
$$

for any $\delta>0$. Letting $\delta \downarrow 0$ and optimizing with respect to $\xi$ and $R$, we obtain (6.17).

\section{References}

[1] F. A. Berezin and M. A. Shubin, The Schrödinger equation. Kluwer Academic Publishers, Dordrecht, 1991. MR 1186643 Zbl 0749.35001

[2] M. S̆. Birman and M. Z. Solomjak, Quantitative analysis in Sobolev imbedding theorems and applications to spectral theory. Amer. Math. Soc., Providence (RI), 1980. MR 0562305 Zbl 0426.46020

[3] Ph. Briet, H. Kovařík, G. Raikov, and E. Soccorsi, Eigenvalue asymptotics in a twisted waveguide. Commun. Partial Differ. Equations 34 (2009), 818-836. MR 2560302 Zbl 1195.35119

[4] Ph. Briet, G. D. Raikov, and E. Soccorsi, Spectral properties of a magnetic quantum Hamiltonian on a strip. Asymptot. Anal. 58 (2008), 127-155. MR 2456460 Zbl 1163.35446

[5] J.-M. Combes and F. Germinet, Edge and impurity effects on quantization of Hall currents. Commun. Math. Phys. 256 (2005), 159-180. MR 2134339 Zbl 1114.81085

[6] J.-M. Combes, P. Hislop, and E. Soccorsi, Edge states for quantum Hall Hamiltonians. In R. Weder (ed.) et al., Mathematical results in quantum mechanics. A conference on QMATH-8, Univ. Nacional Autónoma de México, Taxco, México, December 10-14, 2001. Amer. Math. Soc., Providence (RI), 2002, 69-81. MR 1946017 Zbl 1039.81058

[7] N. Filonov and A. Pushnitski, Spectral asymptotics of Pauli operators and orthogonal polynomials in complex domains. Commun. Math. Phys. 264 (2006), 759-772. MR 2217290 Zbl 1106.81040

[8] U. Grenander and G. Szegő, Toeplitz forms and their applications. University of California Press, Berkeley and Los Angeles (1958). MR 0094840 Zbl 0080.09501

[9] B. C. Hall, Holomorphic methods in analysis and mathematical physics. In S. PérezEsteva (ed.) et al., First summer school in analysis and mathematical physics. Quantization, the Segal-Bargmann transform and semiclassical analysis. Cuernavaca Morelos, Mexico, June 8-18, 1998. Amer. Math. Soc., Providence (RI), 2000, 1-59. MR 1770752 Zbl 0977.46011 
[10] P. Hislop and E. Soccorsi, Edge currents for quantum Hall systems. I. One-edge, unbounded geometries. Rev. Math. Phys. 20 (2008), 71-115. MR 2379247 Zbl 1145.81053

[11] L. Hörmander, Linear partial differential operators. Springer Verlag, New York etc., 1969. MR 0248435 Zbl 0175.39201

[12] M. Kac, W. L. Murdock, and G.Szegő, On the eigenvalues of certain hermitian forms, J. Ration. Mech. Anal. 2 (1953), 767-800. MR 0059482 Zbl 0051.30302

[13] T. Kato, Perturbation theory for linear operators. Springer Verlag, New York etc., 1966. MR 0203473 Zbl 0148.12601

[14] S. V. Khryashchev, On the discrete spectrum of a perturbed periodic Schrödinger operator. Zap. Nauchn. Sem. Leningrad. Otdel. Mat. Inst. Steklov. (LOMI) 190 (1991), 157-162. English transl.: J. Math. Sci. 71 (1994), 2269-2272. MR 1111917 Zbl 0825.47014

[15] M. Melgaard and G. Rozenblum, Eigenvalue asymptotics for weakly perturbed Dirac and Schrödinger operators with constant magnetic fields of full rank. Commun. Partial Differ. Equations 28 (2003), 697-736. MR 1978311 Zbl 1044.35038

[16] P. Miranda and G. Raikov, Discrete spectrum of quantum Hall effect Hamiltonians II. Periodic edge potentials. Preprint 2011. arXiv:1101.1079

[17] M. Persson, Eigenvalue asymptotics of the even-dimensional exterior Landau-Neumann Hamitonian. Adv. Math. Phys. (2009), article ID 873704. MR 2500946 Zbl 1201.81055

[18] A. Pushnitski and G. Rozenblum, Eigenvalue clusters of the Landau Hamiltonian in the exterior of a compact domain. Doc. Math., J. DMV 12 (2007), 569-586. MR 2377242 Zbl 1132.35424

[19] A. Pushnitski and G. Rozenblum, On the spectrum of Bargmann-Toeplitz operators with symbols of a variable sign. To appear in J. Anal. Math.

[20] G. D. Raikov, Eigenvalue asymptotics for the Schrödinger operator with homogeneous magnetic potential and decreasing electric potential. I. Behaviour near the essential spectrum tips. Commun. Partial Differ. Equations 15 (1990), 407-434. MR 1044429 Zbl 0739.35055

[21] G. Raikov, Eigenvalue asymptotics for the Schrödinger operator with perturbed periodic potential. Invent. Math. 110 (1992), 75-93. MR 1181817 Zbl 0801.35095

[22] G. D. Raikov, Eigenvalue asymptotics for the Schrödinger operator in strong constant magnetic fields. Commun. Partial Differ. Equations 23 (1998), 1583-1620. MR 1641780 Zbl 0919.35097

[23] G. D. Raikov and S. Warzel, Quasi-classical versus non-classical spectral asymptotics for magnetic Schrödinger operators with decreasing electric potentials. Rev. Math. Phys. 14 (2002), 1051-1072. MR 1939760 Zbl 1033.81038

[24] M. Reed and B. Simon, Methods of modern mathematical physics I: Functional analysis. Academic Press, New York etc., 1980. MR 0751959 Zbl 0459.46001

[25] M. Reed and B. Simon, Methods of modern mathematical physics. IV: Analysis of operators. Academic Press, New York etc., 1978. MR 0493421 Zbl 0401.47001

[26] G. Rozenblum and G. Tashchiyan, On the spectral properties of the perturbed Landau Hamiltonian. Commun. Partial Differ. Equations 33 (2008), 1048-1081. MR 2424389 Zbl 1158.47029 
[27] K. M. Schmidt, Critical coupling constants and eigenvalue asymptotics of perturbed periodic Sturm-Liouville operators. Commun. Math. Phys. 211 (2000), 465-485. MR 1754525 Zbl 0953.34069

[28] M. A. Shubin, Pseudodifferential operators and spectral theory. Springer Verlag, Berlin, 2001. Second ed. MR $1852334 \mathrm{Zbl} 0980.35180$

[29] L. B. Zelenko, Asymptotic distribution of the eigenvalues in a lacuna of the continuous spectrum of a perturbed Hill operator. Mat. Zametki 20 (1976), 341-350. English transl.: Math. Notes 20(1976) (1977), 750-755. MR 0430404 Zbl 0357.34020

Received September 13, 2010; revised February 7, 2011

Vincent Bruneau, Université Bordeaux I, Institut de Mathématiques de Bordeaux,

UMR CNRS 5251, 351, Cours de la Libération, 33405 Talence, France

E-mail: vbruneau@math.u-bordeaux1.fr

Pablo Miranda, Departamento de Matemáticas, Facultad de Ciencias,

Universidad de Chile, Las Palmeras 3425, Santiago de Chile, Chile

E-mail: pmirandar@ug.uchile.cl

Georgi Raikov, Departamento de Matemáticas, Facultad de Matemáticas,

Pontificia Universidad Católica de Chile, Vicuña Mackenna 4860,

Santiago de Chile, Chile

E-mail: graikov@mat.puc.cl 